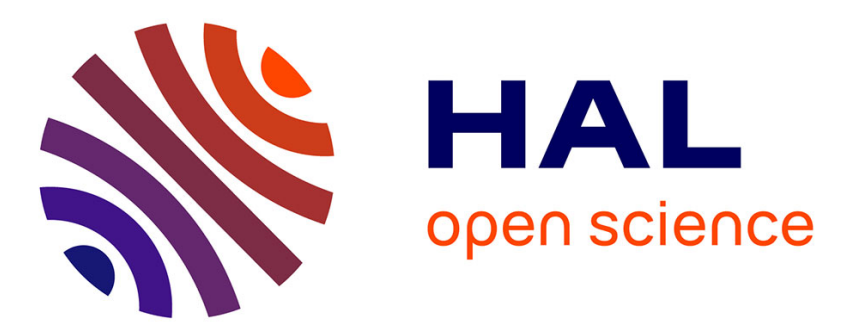

\title{
Prevention efforts, insurance demand and price incentives under coherent risk measures
}

Sarah Bensalem, Nicolás Hernández Santibáñez, Nabil Kazi-Tani

\section{To cite this version:}

Sarah Bensalem, Nicolás Hernández Santibáñez, Nabil Kazi-Tani. Prevention efforts, insurance demand and price incentives under coherent risk measures. 2019. hal-01983433

\section{HAL Id: hal-01983433 \\ https://hal.science/hal-01983433}

Preprint submitted on 16 Jan 2019

HAL is a multi-disciplinary open access archive for the deposit and dissemination of scientific research documents, whether they are published or not. The documents may come from teaching and research institutions in France or abroad, or from public or private research centers.
L'archive ouverte pluridisciplinaire HAL, est destinée au dépôt et à la diffusion de documents scientifiques de niveau recherche, publiés ou non, émanant des établissements d'enseignement et de recherche français ou étrangers, des laboratoires publics ou privés. 


\title{
Prevention efforts, insurance demand and price incentives under coherent risk measures
}

\author{
Sarah Bensalem * $\quad$ Nicolás Hernández Santibáñez ${ }^{\dagger} \quad$ Nabil Kazi-Tani ${ }^{\ddagger}$ \\ January 9, 2019
}

\begin{abstract}
This paper studies an equilibrium model between an insurance buyer and an insurance seller, where both parties' risk preferences are given by convex risk measures. The interaction is modeled through a Stackelberg type game, where the insurance seller plays first by offering prices, in the form of safety loadings. Then the insurance buyer chooses his optimal proportional insurance share and his optimal prevention effort in order to minimize his risk measure. The loss distribution is given by a family of stochastically ordered probability measures, indexed by the prevention effort. We give special attention to the problems of self-insurance and self-protection. We prove that the formulated game admits a unique equilibrium, that we can explicitly solve by further specifying the agents criteria and the loss distribution. In self-insurance, we consider also an adverse selection setting, where the type of the insurance buyers is given by his loss probability, and study the screening and shutdown contracts. Finally, we provide case studies in which we explicitly apply our theoretical results.
\end{abstract}

Key words: Prevention, Self-insurance, Self-protection, Coherent risk measures, Stackelberg game.

AMS 2010 subject classification: 91B06, 91B30, 91A05.

JEL classification: C72, D61, D81, D86, G22.

\section{Introduction}

The fact that rational agents undertake actions to lower their risk exposure is now standard in economic analysis. Insurance buyers can try to reduce the size of the potential claims that they face (self-insurance) or reduce the probability of a claim arrival (self-protection).

In the particular case of health insurance, the increased knowledge of individual health state, through connected devices for instance, make the efforts of risk reduction easier. Insurers themselves start to incentivize prevention efforts: the companies John Hancock or Aetna decided in 2018 to offer connected watches or bracelets to their clients, in exchange for their commitment to a physical activity program, whose progress is measured by the connected device.

The first systematic study of prevention effort, in the form of self-insurance or self-protection, was made in the seminal paper of Erlich and Becker [16]. Their comparative statics analysis showed that the following two conclusions hold true:

\footnotetext{
*ISFA, Université Lyon 1, 50 Avenue Tony Garnier, 69007 Lyon, France. sarah.bensalem@univ-lyon1.fr,

${ }^{\dagger}$ Department of Mathematics, Univerisity of Michigan, nihernan@umich.edu.

${ }^{\ddagger}$ ISFA, Université Lyon 1, 50 Avenue Tony Garnier, 69007 Lyon, France. nabil.kazi-tani@univ-lyon1.fr.
} 
(A) Market insurance and self-insurance are substitutes, in the sense that an increased insurance price implies more prevention effort to reduce the losses size.

(B) Market insurance and self-protection can be complements, in the sense that a more expensive market insurance entails less effort to reduce the probability of losses.

Since then, several papers tested the robustness of these results to various changes in the model (see the paper [9] and the literature below for a short overview). The aim of this paper is to contribute to this line of research in at least three aspects: first, we model the interaction between the insurance buyer and seller as a game and we construct an equilibrium consisting of optimal prevention and insurance parameters for the buyer and optimal price strategy for the seller. Second, as criteria for both market participants, we take general coherent risk measures in the sense of [2]. And last, the impact of the prevention effort is modeled via a family of stochastically ordered probability measures which are indexed by the prevention effort: this allows to deal with self-insurance and self-protection as particular cases, and to give sufficient conditions so that the conclusions (A) and (B) hold true. Let us now describe each of these aspects and the related assumptions.

\section{Summary of the approach.}

A Stackelberg game. The way that we formulate the problem is inspired by contract theory, and in particular by the Principal-Agent problem. Indeed, we consider the insurance buyer as the Agent, whose action is given by a vector $(\alpha, e)$, where $\alpha$ is the proportion of losses paid by the insurer and $e$ is the effort made by the Agent to reduce his risk. Throughout the paper, we will sometimes refer to the insurance buyer as the Agent, and to the insurance seller as the Principal. The contract proposed by the Principal is completely summarized in the price offered for the cover $\alpha$, price which is given in the form of a safety loading (equation (3.1)). The effort $e$ will only impact the losses distribution, and not the losses outcome directly. As a consequence, the choice $\alpha$ will be directly observable by the Principal, whereas the effort action $e$ will be unobservable. This unobservable action case is referred to as the moral hazard situation ([37],[24],[38]). The problem that we consider is a one period model. Static Principal Agent problems involving moral hazard are known to be difficult to solve (see Chapter 2 in [12] or the articles [38, 25] for a discussion on the relaxed formulations of the problem), we are however able to completely solve the Agent's problem in the particular case that we deal with, thanks to the singular form of the optimal coverage $\alpha$, which will be equal to either 0 or 1 . Then, the remaining optimization in the variable $e$ will be non convex, but still solvable by analyzing the problem "locally" and then carefully pasting the solutions together (subsections 3.2 and 3.3).

As already noticed and discussed in [14], the corner type solution for the optimal coverage mainly comes from the positive homogeneity of the criteria (see Definition 2.1) and the linearity of the insurance contract. The linearity of the insurance contract is not a restrictive assumption: it corresponds to some contracts found in practice, in particular in health insurance. It is also extremely common in reinsurance, where this type of contract is referred to as "quota-share". Notice that our analysis readily extends to the relation between an insurer (this time the Agent) and a reinsurer (the Principal), where the effort of the agent in that case consists for instance in underwriting policy control, or in prevention campaigns for the clients.

Risk Measures. In standard contract theory literature (see [37, 24, 38, 39, 12, 10]), the formal optimization problems consist in expected utility maximization. This is also the case in [16] and in most of the papers that followed ([9]). In the present paper however, the criterion of both the Agent and the Principal will be given by a law invariant coherent risk measure (see Definition 2.1). Risk measures are a popular risk assessment tool, in particular because of the associated axiomatic analysis and its interpretation $([2,21])$. It is a common 
tool in insurance and reinsurance practice, since it appears in the european regulation texts: indeed, the quantile function, also called Value-at-Risk, is the risk measure that europeans insurers and reinsurers have to compute, according to the Solvency II rules. Notice that the quantile risk measure is not coherent (which was one of the initial motivations of [2]), and it is quite often in practice compared to the Average Valueat-Risk (AVaR), also called Tail Value-at-Risk, or Expected Shortfall (see [20] for the definitions, and the differences between these measures). AVaR is one example of coherent risk measure, which is also a distortion risk measure (see (2.1)).

The results obtained in this paper are valid if the risk measures used by the Agent and the Principal are law invariant and coherent. This class of risk measures is more general than the class of distortion risk measures. We give in Example 2.1 a class of law invariant coherent risk measures, which are not distortion risk measures. Notice that if $\rho$ is a coherent risk measure, then $U(X):=-\rho(-X)$ defines a monetary utility function. Our results can then be recast in terms of law invariant monetary utility functions, but we state them in the language of risk measures, which is closer to actuarial practice. The general risk sharing problem has been solved in [28], when economic agents are law invariant monetary utility maximizers, and in [35] in the case of quasiconvex risk measures. As showed in [4] and [28], the resolution of the risk sharing problem in the monetary case reduces to the inf-convolution of the given risk measures. In our case however, since the agent's effort is not observable by the Principal, we have to follow another approach, mainly the backward induction, which consists in solving the Agent's problem first and then solving the Principal's problem knowing the optimal responses of the Agent. This is doable here since, contrary to the setting in [4] and [28], we fix the form of insurance contracts, which are linear. We refer the reader to [20,17] for more details on risk measures and insurance applications.

Controlling the distributions. Let us now discuss the type of loss distributions that we consider. In the literature on the arbitrage between market insurance, self-insurance and self-protection, the losses distributions are discrete, concentrated on two points: the insured suffers a loss $L>0$ with probability $p$ or no loss with probability $1-p$, where $p \in(0,1)$. In our setting (see Section 2 for more details), we will consider a family of random variables $\left(X_{e}\right)_{e \in(0,+\infty)}$, indexed by a prevention effort parameter $e$, and such that the distributions of $\left(X_{e}\right)_{e \in(0,+\infty)}$ forms a family of probability measures which is decreasing for the first order stochastic dominance (denoted FSD for simplicity), in the sense that

$$
e_{1}<e_{2} \Longrightarrow E\left[f\left(X_{e_{1}}\right)\right]>E\left[f\left(X_{e_{2}}\right)\right],
$$

for every non-decreasing function $f: \mathbb{R} \rightarrow \mathbb{R}$. In other words, an increase of the effort lowers the dangerousness of the loss, where the dangerousness is measured by the first order stochastic dominance. We will write $X \underset{\text { (mon) }}{\leq} Y$ to say that $X$ is dominated by $Y$ for the FSD, also called the monotone order. We will use the following property of the FSD (see Theorem 2.68 in [20])

$$
X \underset{(\text { mon })}{\leq} Y \Longleftrightarrow \bar{q}_{X}(u) \leq \bar{q}_{Y}(u), \quad \forall u \in(0,1),
$$

where $\bar{q}_{X}$ denotes the tail quantile function associated to the distribution of $X$. Since we will use a family of distributions, indexed by some prevention effort, we will assume that the effort impacts the tail quantile of the loss distribution, in such a way that the marginal impact will be decreasing. Notice that in (1.1), we do not make any convexity nor concavity assumption on $f$, this means that the situation in which the distributions of $\left(X_{e}\right)_{e \in(0,+\infty)}$ are decreasing for the monotone convex order is included in our analysis. 
Using this framework, we are able te deal with self-insurance and self-protection in a unified way. In particular, we provide sufficient conditions for the statements (A) and (B) to hold true, as well as simple counter-examples for $(\mathrm{A})$ and $(\mathrm{B})$.

We give several explicit examples of probability distributions, which are in line with standard actuarial practice. We analyze in more details the case where, for every $e, X_{e}$ has a distribution given by a mixing between a Dirac mass at 0 and a Pareto distribution with some parameter $k(e)$ depending of the effort. For more details on standard actuarial assumptions, we refer the reader to [6].

\section{Related Literature.}

As already mentioned above, there is a large literature on self-insurance and self-protection, and the very brief overview that we give here is by no means exhaustive. We refer the interested reader to [22], [15] (Chapter 9) and to the paper [9], which provides a survey on the economics of prevention.

Most of the literature focuses on the case where the loss distribution concentrates on just two points, 0 (no loss) and some loss level $L>0$. Some contributions already extended that simple framework: [26], [40], [29], examine the effects of agents' risk preferences on their optimal effort, for more general loss distributions, in particular for distributions satisfying the so-called single-crossing property. This property is also used in the paper [3], that provides sufficient conditions on the utility functions and on probability distributions, allowing to obtain comparative statics results.

In the case where the agent is subject to both self-insurance and self-protection activities, [33] studied the case where the loss distribution concentrates on $N$ points, with $N \geq 2$. The author showed that even if the marginal reduction of a loss dominates the marginal increase of the effort's cost, more risk averse agents do not necessarily exert more effort: he provides a sufficient condition on the probability distribution for this result to hold true, this condition is comparable to the one we obtain in subsection 5.2 for statement (A) to hold true. [36] analyzes the interaction between self-protection activities, prudence and risk aversion, under the assumption that a change in the level of self-protection is not mean preserving, but rather mean utility preserving, for any utility function. [8] considers random vectors instead of one-dimensional random variables. The marginal distributions are still bi-variate, and the effect of the dependence structure between the marginals on prevention decisions is studied.

In all these papers, agents maximize their expected utility. Several authors studied the robustness of assertions (A) and (B), or the relationship between risk aversion and prevention efforts, when agents use other criteria. [30] considered the class of Rank Dependent Expected Utilities (RDEU), in which agents can distort the probabilities, i.e. over or underweigh certain loss probabilities. In the language of risk measures, in the RDEU framework, an agent facing a risk $X$ minimizes the quantity $\rho(u(X))$ where $\rho$ is a distortion risk measure (see (2.1)) and $u$ a non-decreasing and concave utility function. It is shown in [30] that more risk averse agents exert more self-insurance effort. In [18], self-protection activities in a health insurance context are studied, in a RDEU framework, with non concave distortions. When RDEU is considered in the particular case of $u(x)=x$, we obtain the so-called Yaari's Dual Theory (DT). [7] analyzed the interaction between market insurance, self-insurance, and self-protection in the DT framework, and proved that the main results of [16] carry over to that case. Let us mention the papers [32] and [5], that focus on the relation between risk aversion and the Willingness to Pay (WTP) for general risk preferences (RDEU in the case of [5]). 


\section{Main contributions.}

The current paper extends the problems of self-insurance and self-protection to the case of a Stackelberg game between the insurance buyer and seller, under law invariant coherent risk measures, and in a situation of moral hazard. We prove that the formulated game always admits an equilibrium that we characterize.

The focus lies on the impact of an increased price on optimal insurance demand and prevention effort. We give emphasis to the fact that whether statements (A) and (B) hold true or not essentially depends on the relative impact of the effort on the losses distribution through the risk measure and on the price (Assumptions 3.1 and 3.2).

The methodology described in this paper can be applied to a large class of insurance claim distributions. To illustrate this, we analyze in detail the case of the Pareto distribution, which is standard in actuarial science and we give some insight about the case of discrete distributions, which is standard in insurance economics.

\section{Structure of the paper}

Section 2 discusses the problem and our main assumptions. In Section 3, we completely solve the buyer problem. Section 4 is dedicated to the seller problem and Section 5 illustrates the proposed methodology in particular cases, where we specify the claim distribution family and the class of risk measures. Finally, we show in the Appendix that the form of probability distribution that we consider can be used as a proxy for the Compound Poisson distribution, which is common in actuarial models.

\section{Model and Assumptions}

Let $(\Omega, \mathbb{F}, \mathbb{P})$ be a fixed probability space. For a given random variable $X, \bar{F}_{X}$ denotes its survival function defined by $\bar{F}_{X}(x):=\mathbb{P}(X>x)$ and $\bar{q}_{X}$ denotes its tail quantile function, defined as the inverse of $\bar{F}_{X}$ :

$$
\bar{q}_{X}(u):=\inf \left\{x \in \mathbb{R} \mid \bar{F}_{X}(x) \leq u\right\} .
$$

We assume that the insured can make some effort, modeled by a parameter $e \in \mathbb{R}^{+}$, in order to reduce its losses, or the probability to suffer losses. Our assumptions allow us to deal with self-insurance and selfprotection in a unified way, using stochastic orders. Let us introduce the probability distributions of insurance claims in more details.

The losses distributions. Let $\left(X_{e}\right)_{e \in[0,+\infty)}$ be a family of non-negative random variables, representing losses, such that the distributions $\mu_{e}$ of $X_{e}$ are decreasing for the FSD. In particular, this implies that for each $u \in(0,1)$, the function $e \mapsto \bar{q}_{X_{e}}(u)$ is non-increasing. To simplify the notations, we will write $Q(u, e):=\bar{q}_{X_{e}}(u)$. We also assume that the marginal impact of the effort is non-decreasing.

Assumption 2.1. For every $u \in(0,1), e \mapsto Q(u, e)$ is a convex function.

The objectives. We choose to model the interaction between the insurance buyer, that we will refer to as IB, and the insurance seller, called IS, as a game in a sequential form: IS plays first by offering prices, then IB plays second by choosing his optimal insurance cover and his optimal effort parameter. We assume that both players minimize a law invariant coherent risk measure, whose definition is recalled below for completeness.

Definition 2.1. $\rho: L^{1} \rightarrow \mathbb{R}$ is a law invariant coherent risk measure if

1. $\rho$ is monotone, in the sense that $X \leq Y$ almost surely implies that $\rho(X) \leq \rho(Y)$. 
2. $\rho$ is cash-additive: $\forall m \in \mathbb{R}, \rho(X+m)=\rho(X)+m$.

3. $\rho$ is convex: For each $X$ and $Y$ in $L^{1}$ and for all $\lambda \in[0,1], \rho(\lambda X+(1-\lambda) Y) \leq \lambda \rho(X)+(1-\lambda) \rho(Y)$.

4. $\rho$ is positively homogeneous: $\forall \lambda \in \mathbb{R}^{+}, \rho(\lambda X)=\lambda \rho(X)$.

5. $\rho$ is law-invariant: $X \stackrel{d}{=} Y$ implies that $\rho(X)=\rho(Y)$, where $\stackrel{d}{=}$ denotes equality in distribution.

If $\rho$ satisfies conditions 1. to 4 . in Definition 2.1, then it is called a coherent risk measure, as introduced in [2]. For more details and properties of risk measures, we refer the reader to [20] (Chapter 4). An important class of law-invariant coherent risk measures is given by the distortion risk measures, defined by

$$
\rho(X):=\int_{0}^{1} \bar{q}_{X}(u) d \psi(u)
$$

where $\psi$ is a distortion function, i.e. a non-decreasing function from $[0,1]$ to $[0,1]$ such that $\psi(0)=0$ and $\psi(1)=1$. If the probability space $(\Omega, \mathcal{F}, \mathbb{P})$ has no atoms, then one can prove that a risk measure of the form (2.1) is convex if and only if the distortion function $\psi$ is concave.

Example 2.1. Let us give an example of coherent risk measure which is not a distortion risk measure:

$$
\rho(X):=\mathbb{E}[X]+\delta \mathbb{E}\left[(\mathbb{E}[X]-X)_{-}^{p}\right]^{1 / p}, \quad \delta \in(0,1), \quad 1<p<+\infty,
$$

satisfies all properties of Definition 2.1, and cannot be written in the form (2.1). We refer the reader to [28] (Section 3.4) for more details on the so-called semi-deviation risk measure.

Lemma 2.1. Let $\rho$ be a risk measure satisfying the properties 1,2,3 and 5 in Definition 2.1. If the distributions of $\left(X_{e}\right)_{e \in[0,+\infty)}$ satisfy Assumption 2.1, then the function $e \mapsto \rho\left(X_{e}\right)$ is convex.

Proof. Assume first that $\rho$ takes the form given in (2.1), which we can write as

$$
\rho\left(X_{e}\right)=\int_{0}^{1} Q(u, e) d \psi(u)
$$

Since $e \mapsto Q(u, e)$ is convex, then $e \mapsto \int_{0}^{1} Q(u, e) d \psi(u)$ is also convex. Now, if $\rho$ is a general risk measure satisfying 1,2,3 and 5 in Definition 2.1, by Theorem 4.62 and Corollary 4.78 in [19], we have the following Legendre-Fenchel type representation:

$$
\rho\left(X_{e}\right)=\sup _{\psi}\left\{\int_{0}^{1} \bar{q}_{X_{e}}(u) d \psi(u)-\gamma(\psi)\right\},
$$

where the supremum is taken over all concave distortion functions $\psi$ and where

$$
\gamma(\psi)=\sup _{Y \in A_{\rho}}\left\{\int_{0}^{1} \bar{q}_{Y}(u) d \psi(u)\right\},
$$

with $A_{\rho}:=\left\{Y \in L^{1} \mid \rho(Y) \leq 0\right\}$. Notice that to be able to apply Corollary 4.78 in [20], we need a continuity property of $\rho$ (the so-called Fatou property), which is automatically satisfied by Theorem 2.1 in [27]. By (2.2), the function $e \mapsto \rho\left(X_{e}\right)$ is a supremum of convex functionals, and hence it is also convex.

Remark 2.1. The previous Lemma entails that $e \mapsto \rho\left(X_{e}\right)$ is continuous. From representation $(2.2)$, the map $e \mapsto \rho\left(X_{e}\right)$ is also non-increasing and we have $\rho\left(X_{e}\right) \geq \mathbb{E}\left[X_{e}\right] \geq 0$.

Remark 2.2. For the particular distortion function $\psi(u)=u$, the risk measure defined in (2.1) reduces to $\rho(X)=\mathbb{E}(X)$. Then by Lemma 2.1, we have that $e \mapsto \mathbb{E}\left(X_{e}\right)$ is a continuous, non-increasing and convex function. 
The sequential game. As mentioned above, we model the interaction between IB and IS through a game, where IS plays first and IB plays second. We solve this game using a backward induction argument.

We assume that IB has an initial wealth denoted $\omega_{0}$ and that he is facing a risk with loss $X_{e}$ for which he subscribes to a proportional insurance contract with IS. In this insurance program, he chooses his level of insurance denoted $\alpha \in[0,1]$ and he pays a premium denoted $\Pi$. The prevention effort $e \in \mathbb{R}^{+}$made by IB has a monetary cost $c(e)$, where $c$ is a non-decreasing and strictly convex function. ${ }^{1}$ We suppose that IB uses a law-invariant and coherent risk measure $\rho_{1}$. The goal of IB is to minimize the risk measure associated to his total loss, which is given by:

$$
L_{1}(\alpha, e):=\rho_{1}\left((1-\alpha) X_{e}+\Pi\left(\alpha X_{e}\right)+c(e)-\omega_{0}\right) .
$$

Using the positive homogeneity and cash-additivity properties of $\rho$, we obtain that IB's objective function simplifies to

$$
L_{1}(\alpha, e)=(1-\alpha) \rho_{1}\left(X_{e}\right)+\Pi\left(\alpha X_{e}\right)+c(e)-\omega_{0} .
$$

Therefore IB wants to solve the following optimization problem:

$$
\inf _{(\alpha, e) \in[0,1] \times(0, \infty)} L_{1}(\alpha, e) .
$$

Remark 2.3. From this point forward, we consider the initial wealth $\omega_{0}$ to be equal to 0. This is without loss of generality, by the cash-additivity of $\rho_{1}$.

On the other hand, IS wants to find the best premium for the insurance coverage that IB is willing to accept. We suppose that IS uses a law-invariant and coherent risk measure $\rho_{2}$ and her goal is to minimize her risk measure associated to her loss, given by

$$
L_{2}(\Pi):=\rho_{2}\left(\alpha^{\star} X_{e^{\star}}-\Pi\left(\alpha^{\star} X_{e^{\star}}\right)\right),
$$

where $\alpha^{\star}$ and $e^{\star}$ are functions of the premium $\Pi$ and correspond to the insurance demanded by IB and the effort he performs under it. Therefore, IS solves the following optimization problem

$$
\inf _{\Pi} L_{2}(\Pi),
$$

where the set of premiums over which IS optimizes will be specified in the next section.

\section{The insurance buyer's optimization problem}

\subsection{Optimal insurance cover}

We start the resolution of IB's problem by studying his optimal insurance coverage. In the literature this coverage is either equal to 0 or 1 (see [7], [34], [41], or see [14] for empirical results). In the following Proposition, we give a sufficient condition on the pricing functional $\Pi$ under which the optimal value for $\alpha$ is either 0 or 1.

Proposition 3.1. Assume that $\Pi$ is positively homogeneous

$$
\Pi(\lambda X)=\lambda \Pi(X), \quad \forall \lambda \geq 0,
$$

then the optimal insurance coverage $\alpha^{\star}$ only takes the values 0 or 1.

\footnotetext{
${ }^{1}$ If $\mathrm{c}$ is merely convex, all our analysis holds true by choosing the smallest effort realizing the minimum when needed.
} 
Proof. By our assumptions, we get that the objective function $L_{1}$ is linear in $\alpha$, since

$$
L_{1}(\alpha, e)=(1-\alpha) \rho_{1}\left(X_{e}\right)+\alpha \Pi\left(X_{e}\right)+c(e) .
$$

So the optimal insurance demand only depends on the sign of $\Pi\left(X_{e}\right)-\rho_{1}\left(X_{e}\right)$, and is given by

$$
\alpha^{\star}(e)= \begin{cases}1 & \text { if } e \in \mathcal{I} \\ 0 & \text { if } e \in \mathcal{N}:=\mathcal{I}^{c},\end{cases}
$$

where $\mathcal{I}:=\left\{e \in \mathbb{R}^{+} \mid \Pi\left(X_{e}\right) \leq \rho_{1}\left(X_{e}\right)\right\}$.

In other words, the optimal coverage will be the full insurance coverage $\alpha^{\star}=1$ when the price asked by the insurer is lower than the risk associated to the claims distribution, and it will be 0 otherwise.

In the actuarial science literature, various premium functionals are analyzed (see [31] or [13]). One standard example is the safety loading:

$$
\Pi(X):=(1+\theta) \mathbb{E}[X]
$$

where $\theta$ is a non-negative parameter. In this case, $\mathcal{I}:=\left\{e \in \mathbb{R}^{+} \mid 1+\theta \leq \frac{\rho_{1}\left(X_{e}\right)}{\mathbb{E}\left[X_{e}\right]}\right\}$. For $\theta=0$, this is the so-called pure premium principle.

Another example is the standard deviation principle:

$$
\Pi(X):=\mathbb{E}[X]+\gamma \sigma_{X}
$$

where $\sigma_{X}$ denotes the standard deviation of $X$ and $\gamma$ is a non-negative parameter. In this case we have $\mathcal{I}:=\left\{e \in \mathbb{R}^{+} \mid \gamma \leq \frac{\rho_{1}\left(X_{e}\right)-E\left[X_{e}\right]}{\sigma_{X_{e}}}\right\}$.

Remark 3.1. The safety loading and the standard deviation principles are two ways to compute premiums that satisfy the positive homegeneity property given in Proposition 3.1. If this property is not satisfied, then one can find an interior solution $\alpha^{*} \in(0,1)$ for the optimal insurance coverage. In the paper [14], the authors avoid corner solutions in two ways: by considering a non linear contract with a deductible, or by introducing a source of background risk. In our case, we could consider a premium functional $\Pi$ which is not homogeneous, and introduce interior solutions this way, even in the case of a single insurable source of risk. An example of such functional $\Pi$ is the variance principle $\Pi(X):=\mathbb{E}[X]+\beta V(X)$.

Remark 3.2. Notice that for Proposition 3.1 to hold true, we only need $\rho_{1}$ to satisfy the properties 2 and 4 of cash-additivity and positive homogeneity of Definition 2.1.

Throughout the rest of the paper, we assume for simplicity that $\Pi(X)=(1+\theta) \mathbb{E}[X]$, with $\theta \geq 0$, but our results can be generalized to other insurance pricing functionals. Recall that IB demands (full) insurance, that is $\alpha^{\star}(e)=1$, if $e \in \mathcal{I}$ and IB demands no insurance, that is $\alpha^{\star}(e)=0$ if $e \in \mathcal{N}$, where

$$
\mathcal{I}:=\left\{e \in \mathbb{R}^{+} \mid 1+\theta \leq \frac{\rho_{1}\left(X_{e}\right)}{\mathbb{E}\left[X_{e}\right]}\right\} .
$$

Let us define then the following function $G$ and state our main assumption on it, that says how the effort impacts the risk and the price of insurance (represented by the expectation, given the safety loading premium).

$$
G(e):=\frac{\rho_{1}\left(X_{e}\right)}{\mathbb{E}\left[X_{e}\right]}
$$


In this paper we assume the function $G$ is monotonic, which gives two different cases that we study separately. We first assume that $G$ is non-increasing, which is a natural economic assumption, since it means that an increased effort has a bigger impact on the risk than it has on the price: we analyze the self-insurance problem under this assumption. Then, in subsection 3.3 we assume that $G$ is non-decreasing: we prove that this assumption is always satisfied in the self-protection case. In both cases, we will solve completely the problem of IB and discuss the veracity of statements (A) and (B) mentioned in the Introduction.

\subsection{Non-increasing $G$}

As discussed in Section 2, suppose that the agent can exert an effort to reduce the risk that he is exposed to. In this subsection, we assume that an increased effort has a bigger impact on the risk than it has on the price. We will prove next that for any model satisfying Assumption 3.1 below, the statement (A) of the Introduction holds true, i.e. less market insurance translates into more prevention effort.

Assumption 3.1. The function $G$ defined in (3.2) is non-increasing, or equivalently

$$
e_{1} \leq e_{2} \Longrightarrow \frac{\rho_{1}\left(X_{e_{2}}\right)}{\rho_{1}\left(X_{e_{1}}\right)} \leq \frac{\mathbb{E}\left[X_{e_{2}}\right]}{\mathbb{E}\left[X_{e_{1}}\right]}
$$

We already know that the functions $e \mapsto \mathbb{E}\left[X_{e}\right]$ and $e \mapsto \rho\left(X_{e}\right)$ are non-increasing and convex. The assumption above asserts that the effort impact is bigger on the risk measure $\rho_{1}$ than it is on the expectation. This assumption is satisfied in various models of self-insurance, which is the main example we have in mind, although the analysis we develop and our results are more general.

Example 3.1. Consider the distribution of the loss random variable $X_{e}$ given by

$$
P_{X_{e}}:=(1-p) \delta_{\{0\}}+p P_{Y_{e}},
$$

where $0<p<1, P_{Y_{e}}$ denotes the distribution (controlled by IB) of a positive random variable, and $\delta_{\{0\}}$ is the Dirac mass at 0. As shown in Section 5.1, when the random variables $Y_{e}$ are Pareto distributed, Assumption 3.1 is satisfied. We also mention the cases where the random variables $Y_{e}$ have a Fréchet, Weibull or Log-Normal distribution, as other standard examples in which Assumption 3.1 is satisfied.

We proceed now to solve the problem (2.4) of IB, consisting in the minimization of the functional $L_{1}$. For fixed $e$, we already solved the problem of minimizing $L_{1}$ in alpha. For fixed $\alpha$, we know from Lemma 2.1 and Remark 2.2 that

$$
L_{1}(\alpha, e)=(1-\alpha) \rho_{1}\left(X_{e}\right)+(1+\theta) \mathbb{E}[X]+c(e),
$$

is convex, as the sum of three convex functions. Notice now that the function $e \mapsto L_{1}\left(\alpha^{\star}(e), e\right)$ is not convex, so its minimization is not straightforward. However, we can take advantage of the fact that $\alpha^{\star}$ only takes the values 0 and 1 to study $L_{1}\left(\alpha^{\star}(e), e\right)$ separately on the sets $\mathcal{N}$ and $\mathcal{I}$, on which it is a convex function, and then compare the local minima on $\mathcal{N}$ and $\mathcal{I}$ to get a global minimum.

Define $L_{\mathcal{N}}$ and $L_{\mathcal{I}}^{\theta}$ as follows, for $e \in \mathbb{R}^{+}$,

$$
\begin{aligned}
L_{\mathcal{N}}(e) & :=\rho\left(X_{e}\right)+c(e), \\
L_{\mathcal{I}}^{\theta}(e) & :=(1+\theta) \mathbb{E}\left[X_{e}\right]+c(e) .
\end{aligned}
$$

$L_{\mathcal{N}}$ and $L_{\mathcal{I}}^{\theta}$ are convex and such that

$$
L_{1}\left(\alpha^{\star}(e), e\right)= \begin{cases}L_{\mathcal{N}}(e), & \text { if } e \in \mathcal{N}, \\ L_{\mathcal{I}}^{\theta}(e), & \text { if } e \in \mathcal{I} .\end{cases}
$$


Lemma 3.1. There exists $e_{\theta} \in \mathbb{R}^{+}$such that $\mathcal{N}$ and $\mathcal{I}$ are two intervals of the form

$$
\begin{aligned}
\mathcal{N} & =\left(e_{\theta},+\infty\right), \\
\mathcal{I} & =\left[0, e_{\theta}\right] .
\end{aligned}
$$

Proof. We already know from Lemma 2.1 that both functions $e \mapsto \rho_{1}\left(X_{e}\right)$ and $e \mapsto \mathbb{E}\left[X_{e}\right]$ are continuous. Also, since $X_{e}$ has finite expectation, $G(e)=\frac{\rho_{1}\left(X_{e}\right)}{\mathbb{E}\left[X_{e}\right]}$ is continuous and non-increasing. We can define its right continuous inverse $G^{-1}(y):=\inf \left\{e \in \mathbb{R}^{+} \mid G(e)<y\right\}$ and

$$
e_{\theta}:=G^{-1}(1+\theta)
$$

Notice that $G\left(e_{\theta}\right)=1+\theta$, since $G$ is continuous. Then by definition, for every $e \leq e_{\theta}, G(e) \geq 1+\theta$, which means that $e \in \mathcal{I}$ and for every $e>e_{\theta}, G(e)<1+\theta$, which means that $e \in \mathcal{N}$.

Let us now study the function $L_{\mathcal{I}}^{\theta}$. It is a continuous and strictly convex function, that goes to $+\infty$ as $e$ tends to $+\infty .^{2}$ So it has a unique minimizer on $\mathbb{R}^{+}$that we denote $e_{I}(\theta)$. The following Proposition identifies the local minimizer of $L_{\mathcal{I}}^{\theta}$ on the set $\mathcal{I}$.

Proposition 3.2. Suppose that $e \mapsto \mathbb{E}\left[X_{e}\right]$ and $e \mapsto c(e)$ are differentiable. There exist constants $\theta_{0}<\theta_{I}$ such that

(a) $\theta \leq \theta_{I} \Longrightarrow \underset{\mathcal{I}}{\operatorname{argmin}} L_{\mathcal{I}}^{\theta}=\left\{e_{I}(\theta)\right\}$.

(b) $\theta_{I}<\theta \Longrightarrow \underset{\mathcal{I}}{\operatorname{argmin}} L_{\mathcal{I}}^{\theta}=\left\{e_{\theta}\right\}$.

Moreover $\theta \leq \theta_{0} \Longrightarrow e_{I}(\theta)=0$.

Proof. For simplicity, we will write $E(e):=\mathbb{E}\left[X_{e}\right]$. Since the random variables $\left(X_{e}\right)_{e \in \mathbb{R}^{+}}$have finite expectation, the function $L_{\mathcal{I}}^{\theta}$ is finite at $e=0$. Its right hand derivative $\frac{d}{d e} L_{\mathcal{I}}^{\theta}(0)$ is well defined and

$$
\frac{d}{d e} L_{\mathcal{I}}^{\theta}(0) \geq 0 \Longleftrightarrow \theta \leq-\frac{c^{\prime}(0)}{E^{\prime}(0)}-1=: \theta_{0}
$$

In that case, $L_{\mathcal{I}}^{\theta}$ is non-decreasing at 0 , and by convexity it is non-decreasing everywhere, so its minimum is attained at $e=0$. Notice that for the constant $\theta_{0}$ to be well defined, we need that $E^{\prime}(0) \neq 0$. If this is not the case, then $\frac{d}{d e} L_{\mathcal{I}}^{\theta}(0)=c^{\prime}(0) \geq 0$ and the function $L_{\mathcal{I}}^{\theta}$ is non-decreasing for any $\theta \in \mathbb{R}$.

We analyze now the case where $\theta>\theta_{0}$. This entails that the function $L_{\mathcal{I}}^{\theta}$ is decreasing at 0 . Therefore, the minimizer $e_{I}(\theta)$ of $L_{\mathcal{I}}^{\theta}$ on $\mathbb{R}^{+}$is an interior point characterized by

$$
1+\theta=-\frac{c^{\prime}\left(e_{I}(\theta)\right)}{E^{\prime}\left(e_{I}(\theta)\right)}
$$

Let us prove that $\theta \mapsto e_{I}(\theta)$ is non-decreasing. Indeed, the function $e \mapsto c^{\prime}(e) \frac{-1}{E^{\prime}(e)}$ is non-decreasing, being the product of two non-negative non-decreasing functions. Since $1+\theta$ is obviously increasing with $\theta,(3.7)$ implies that $e_{I}(\theta)$ is non-decreasing with $\theta$.

\footnotetext{
${ }^{2}$ Since $L_{\mathcal{I}}^{\theta}(e) \geq c(e)$, and $c(e)$ being non-decreasing and strictly convex, we have $\lim _{e \rightarrow+\infty} c(e)=+\infty$.
} 
Notice that since the function $e \mapsto \frac{\rho_{1}\left(X_{e}\right)}{\mathbb{E}\left[X_{e}\right]}$ is non-increasing, $\theta \mapsto e_{\theta}$ is non-increasing. Notice also that since $\frac{\rho_{1}\left(X_{e}\right)}{\mathbb{E}\left[X_{e}\right]} \geq 1$ for every $e$ in $\mathbb{R}^{+}$, when $\theta=0, \mathcal{I}=\mathbb{R}^{+}$and $e_{\theta}$ goes to $+\infty$ when $\theta$ tends to 0 . Thus the following constant $\theta_{I}$ is well defined:

$$
\theta_{I}:=\inf \left\{\theta>0 \mid e_{\theta} \leq e_{I}(\theta)\right\}
$$

Said otherwise, if $\theta$ is greater than $\theta_{I}$, then the global minimizer $e_{I}(\theta)$ lies outside of the set $\mathcal{I}$, and if $\theta$ is lower than $\theta_{I}, e_{I}(\theta) \in \mathcal{I}$. In particular, $\theta \leq \theta_{I}$ implies that the local minimizer of $L_{\mathcal{I}}^{\theta}$ on $\mathcal{I}$ is given by $e_{I}(\theta)$. On the other hand, when $\theta>\theta_{I}$, then the restriction of $L_{\mathcal{I}}^{\theta}$ on $\mathcal{I}$ is non-increasing, and its local minimizer is given by the bound $e_{\theta}$. To conclude the proof, notice that $\theta_{0}<\theta_{I}$, since for $e_{I}(\theta)$ to be greater than $e_{\theta}$ (or even to be greater than 0 ), the function $L_{\mathcal{I}}^{\theta}$ has to be decreasing at 0 .

Let us now study the local minimizer of $L_{\mathcal{N}}$ on the set $\mathcal{N}$. The function $L_{\mathcal{N}}(e)=\rho\left(X_{e}\right)+c(e)$ is non-negative, strictly convex and coercive. Therefore it admits a unique minimizer on $\mathbb{R}^{+}$, that we denote $e_{N}$.

Proposition 3.3. There exists a positive constant $\theta_{N}$ such that

(a) $\theta \leq \theta_{N} \Longrightarrow \underset{\mathcal{N}}{\operatorname{argmin}} L_{\mathcal{N}}=\left\{e_{\theta}\right\}$.

(b) $\theta>\theta_{N} \Longrightarrow \underset{\mathcal{N}}{\operatorname{argmin}} L_{\mathcal{N}}=\left\{e_{N}\right\}$.

Proof. We know that $\theta \mapsto e_{\theta}$ is non-increasing. Define then ${ }^{3}$

$$
\theta_{N}:=\inf \left\{\theta \geq 0 \mid e_{\theta}<e_{N}\right\} .
$$

Then $\theta>\theta_{N}$ implies that $e_{N} \in \mathcal{N}$. If $\theta \leq \theta_{N}$, then $L_{\mathcal{N}}$ is non-decreasing on $\mathcal{N}$, and its minimizer is given by the boundary point $e_{\theta}$.

There remains to determine the global minimum of the function $e \mapsto L_{1}\left(\alpha^{\star}(e), e\right)$ defined in (3.4). This can be done by carefully analyzing the dynamics of $e_{I}(\theta)$ and $e_{\theta}$ as functions of $\theta$ : this is what we do in the proof of the next result.

Theorem 3.1. Let $e^{\star}:=\underset{e}{\operatorname{argmin}} L_{1}\left(\alpha^{\star}(e), e\right)$ be the global minimum of the function $e \mapsto L_{1}\left(\alpha^{\star}(e), e\right)$. There exists a positive constant $\theta_{M}$ such that

(a) $\theta \leq \theta_{M} \Longrightarrow e^{\star}=e_{I}(\theta)$.

(b) $\theta>\theta_{M} \Longrightarrow e^{\star}=e_{N}$.

$\theta_{M}$ represents the maximum value of the safety loading such that the insurance buyer accepts to enter the contract and pay the price given by $\left(1+\theta_{M}\right) \mathbb{E}\left[X_{e_{I}\left(\theta_{M}\right)}\right]$. For $\theta>\theta_{M}$, he chooses not to buy any insurance and exerts the optimal effort $e_{N}$.

To prove Theorem 3.1, we will need the following Lemma.

Lemma 3.2. There does not exist any $\theta \geq 0$ such that

$$
e_{N}<e_{I}(\theta)<e_{\theta}
$$

\footnotetext{
${ }^{3}$ The fact that $\theta_{N}$ is finite is proved in Step 1 of the proof of Lemma 3.2 (see (3.11)).
} 
Proof. Step 1: We first prove that there exists $\theta>0$ such that

$$
e_{\theta} \leq e_{N}
$$

By assumption, the expectation $\mathbb{E}\left[X_{e}\right]$ is finite for every $e \geq 0$. This does not imply the finiteness of the risk measure values $\rho\left(X_{e}\right)$. Thus, if $e \mapsto \rho\left(X_{e}\right)$ is not finite everywhere, we define the minimal effort $e_{\min }$ such that the agent will never exert an effort less or equal to that value, in other words:

$$
e_{\min }:=\inf \left\{e>0 \mid \rho\left(X_{\tilde{e}}\right)<+\infty, \forall \tilde{e}>e\right\} \text {. }
$$

In particular, $\rho\left(X_{e_{\min }}\right)=+\infty$ and $\rho\left(X_{e}\right)<+\infty$ for each $e>e_{\min }$. If $e \mapsto \rho\left(X_{e}\right)$ is finite everywhere, we define $e_{\min }:=0$. Let us show that $e_{\min } \leq e_{N}$. Indeed, if $\rho\left(X_{e_{\min }}\right)=+\infty$, since $e_{N}$ is the global minimizer of $e \mapsto \rho\left(X_{e}\right)+c(e)$, we have $e_{N}>e_{\min }$. If $e_{\min }=0$, the inequality is also satisfied since $e_{N} \geq 0$ by definition. Since, also by definition, $e_{\theta}$ converges to $e_{\min }$ as $\theta$ goes to $+\infty$, we obtain that for a large enough $\theta$, the inequality (3.11) is satisfied.

Step 2: Let us assume the existence of $\tilde{\theta} \geq 0$ satisfying (3.10).

Since the function $\theta \mapsto e_{I}(\theta)$ is non-decreasing and $\theta \mapsto e_{\theta}$ is non-increasing, and by inequality (3.11), we have the existence of $\theta$ larger than $\tilde{\theta}$ such that

$$
e_{N}<e_{\theta}<e_{I}(\theta)
$$

By the definition (3.5) of $e_{\theta}$, we have

$$
\begin{aligned}
& L_{\mathcal{N}}(e) \geq L_{\mathcal{I}}^{\theta}(e), \quad \forall e \leq e_{\theta} \quad \text { and } \\
& L_{\mathcal{N}}(e)<L_{\mathcal{I}}^{\theta}(e), \quad \forall e>e_{\theta} .
\end{aligned}
$$

In particular, using (3.13), we get $L_{\mathcal{N}}\left(e_{N}\right) \geq L_{\mathcal{I}}^{\theta}\left(e_{N}\right)$ and $L_{\mathcal{N}}\left(e_{I}(\theta)\right)<L_{\mathcal{I}}^{\theta}\left(e_{I}(\theta)\right)$, which is impossible since $e_{N}$ and $e_{I}(\theta)$ are the respective global minimizers of $L_{\mathcal{N}}$ and $L_{\mathcal{I}}^{\theta}$. This proves the claim of the Lemma.

Remark 3.3. The economic interpretation of the previous Lemma is quite natural: it says that for prices not too high (safety loadings not too high), the agent cannot be fully insured and exert more effort than when he has no insurance.

Proof. [of Theorem 3.1] On one hand, since $\rho\left(X_{e}\right) \geq \mathbb{E}\left[X_{e}\right]$ for every $e \geq 0, e_{\theta}$ converges to $G^{-1}(1)=+\infty$ as $\theta$ approaches $0^{+}$. On the other hand, notice that $e_{I}(0) \leq e_{N}$, since the reverse inequality is impossible by Lemma 3.2. Recall the definition (3.9) of the constant $\theta_{N}$. So the situation for $\theta$ small enough $\left(\theta \leq \theta_{N}\right)$ is:

$$
e_{I}(\theta) \leq e_{N}<e_{\theta}
$$

Furthermore, by (3.11), there exists $\theta$ large enough $\left(\theta>\theta_{N}\right)$ such that

$$
e_{I}(\theta) \leq e_{\theta} \leq e_{N}
$$

Notice that in the case where there exists $\theta_{J}$ such that $e_{I}(\theta)>e_{N}$ for every $\theta \geq \theta_{J}$, then we have $\theta_{N} \leq \theta_{J}$, since the reverse inequality would imply the existence of $\theta$ such that $e_{N}<e_{I}(\theta)<e_{\theta}$, which is not possible by Lemma 3.2. So the inequalities (3.14) and (3.15) are the only possible dynamics for these effort levels, as functions of $\theta$.

In the case where $\theta \leq \theta_{N}$, both $e_{I}(\theta)$ and $e_{N}$ belong to the set $\mathcal{I}$, so the global minimizer of $L_{1}$ is given by $e_{I}(\theta)$. 
When $\theta>\theta_{N}$, we need to compare $L_{\mathcal{I}}^{\theta}\left(e_{I}(\theta)\right)$ and $L_{\mathcal{N}}\left(e_{N}\right)$. Let us prove that $\theta \mapsto L_{\mathcal{I}}^{\theta}\left(e_{I}(\theta)\right)$ is non-decreasing. To this end, let $0<\theta_{1}<\theta_{2}$, the fact that $e_{I}\left(\theta_{1}\right)$ is the global minimizer of $L_{\mathcal{I}}^{\theta_{1}}$ gives (recall the notation $\left.E(e):=\mathbb{E}\left[X_{e}\right]\right)$ :

$$
\begin{aligned}
L_{\mathcal{I}}^{\theta_{1}}\left(e_{I}\left(\theta_{1}\right)\right)=\left(1+\theta_{1}\right) E\left(e_{I}\left(\theta_{1}\right)\right)+c\left(e_{I}\left(\theta_{1}\right)\right) & \leq\left(1+\theta_{1}\right) E\left(e_{I}\left(\theta_{2}\right)\right)+c\left(e_{I}\left(\theta_{2}\right)\right) \\
& \leq\left(1+\theta_{2}\right) E\left(e_{I}\left(\theta_{2}\right)\right)+c\left(e_{I}\left(\theta_{2}\right)\right)=L_{\mathcal{I}}^{\theta_{2}}\left(e_{I}\left(\theta_{2}\right)\right) .
\end{aligned}
$$

Thus we can define $\theta_{M}$ by

$$
\theta_{M}:=\inf \left\{\theta \geq 0 \mid L_{\mathcal{I}}^{\theta}\left(e_{I}(\theta)\right) \geq L_{\mathcal{N}}\left(e_{N}\right)\right\}
$$

Then the conclusions of the Theorem hold true by definition.

To conclude this section, let us state the following Corollary of Theorem 3.1, which says that in self-insurance statement (A) from the Introduction is always true. An increase in the premium leads to an increase in the effort, as long as the price is attractive for IB. This holds because by Lemma 3.2, the situation where $e_{N}<e_{I}(\theta)<e_{\theta}$ cannot happen.

Corollary 3.1. The optimal effort $e^{\star}(\theta)$ is a non-decreasing function of $\theta$.

Notice that the optimal effort $e^{\star}(\theta)$ is not a continuous function of $\theta$ : it has a jump at the point $\theta=\theta_{M}$, and this function becomes constant (equal to $e_{N}$ ) after $\theta_{M}$.

\subsection{Self-Protection}

In the previous subsection, we made the Assumption 3.1 stating that the impact of an increased effort is bigger on the risk measure than it is on the expectation. This assumption implies that the conclusion (A) of the seminal paper of Ehrlich and Becker [16] is satisfied. We study now the remaining case where the function $G$ is non-decreasing, and prove that in that case, conclusion (B) always holds true. This will be the case for any model of self-protection, in the form (3.16) described below.

Assumption 3.2. The function $G$ defined in (3.2) is non-decreasing, or equivalently

$$
e_{1} \leq e_{2} \Longrightarrow \frac{\rho_{1}\left(X_{e_{2}}\right)}{\rho_{1}\left(X_{e_{1}}\right)} \geq \frac{\mathbb{E}\left[X_{e_{2}}\right]}{\mathbb{E}\left[X_{e_{1}}\right]}
$$

An example of such a situation, is the one in which the insurance buyer controls his loss probability by exerting an effort $e$ on it. More precisely, let the probability distribution $P_{e}$ of the random variable $X_{e}$ take the following form

$$
P_{e}:=(1-p(e)) \delta_{\{0\}}+p(e) P_{Y},
$$

where $e \mapsto p(e)$ is a decreasing function, $P_{Y}$ denotes the distribution of a positive random variable $Y$, and $\delta_{\{0\}}$ is the Dirac mass at 0 . In other words, the loss random variable $X_{e}$ is strictly positive with probability $p(e)$, in which case its value is given by $Y$, and takes the value 0 with probability $1-p(e)$.

Lemma 3.3. Assume that the loss distribution has the form (3.16) and $\rho_{1}$ is a law invariant and coherent risk measure. Then Assumption 3.2 is satisfied. 
Proof. Let us first suppose that $\rho_{1}$ is a distortion risk measure in the form of $(2.1)$, with a concave distortion $\psi$. We get then:

$$
\begin{aligned}
\rho_{1}\left(X_{e}\right) & =\int_{0}^{1} \bar{q}_{X_{e}}(u) d \psi(u), \\
& =\int_{0}^{p(e)} \bar{q}_{Y}\left(\frac{u}{p(e)}\right) d \psi(u), \\
& =p(e) \int_{0}^{1} \bar{q}_{Y}(v) d \psi(p(e) v) d v .
\end{aligned}
$$

Since $\mathbb{E}\left[X_{e}\right]=p(e) \mathbb{E}[Y]$, we have

$$
\frac{\rho_{1}\left(X_{e}\right)}{\mathbb{E}\left[X_{e}\right]}=\frac{\int_{0}^{1} \bar{q}_{Y}(v) d \psi(p(e) v) d v}{\mathbb{E}[Y]} .
$$

The concavity of $\psi$ implies that the function $e \mapsto \frac{\rho_{1}\left(X_{e}\right)}{\mathbb{E}\left[X_{e}\right]}$ is non-decreasing, which in turn implies that Assumption 3.2 is satisfied. In the case where $\rho_{1}$ is a general law invariant coherent risk measure, using the representation (2.2) and the same computations as above, we get:

$$
\frac{\rho_{1}\left(X_{e}\right)}{\mathbb{E}\left[X_{e}\right]}=\frac{\sup _{\psi}\left\{\int_{0}^{1} \bar{q}_{Y}(v) d \psi(p(e) v) d v-\gamma(\psi)\right\}}{\mathbb{E}[Y]},
$$

where the supremum is taken over all concave distortion functions $\psi$, and where $\gamma(\psi)$ is defined in (2.3). Since for any fixed concave $\psi, e \mapsto \int_{0}^{1} \bar{q}_{Y}(v) d \psi(p(e) v) d v$ is non-decreasing, we can conclude that $e \mapsto \frac{\rho_{1}\left(X_{e}\right)}{\mathbb{E}\left[X_{e}\right]}$ is also non-decreasing.

This result says that when only the loss probability is controlled, then the impact of the effort is bigger on the price (the expectation) than it is on the risk. As it will become clear from the next results, this implies that there are situations in which an increase in the insurance price leads to less effort to reduce the loss probability, that is the well known conclusion (B) of [16].

Remark 3.4. Given the particular form (3.16) of probability distributions corresponding to self-protection, Assumption 2.1, which ensures that $e \mapsto \rho_{1}\left(X_{e}\right)$ is convex, says in this setting that $e \mapsto \bar{q}_{Y}\left(\frac{u}{p(e)}\right), 0 \leq u \leq p(e)$, is convex. This is the case, for example, if $u \mapsto \bar{q}_{Y}(u)$ and $e \mapsto p(e)$ are both concave.

In the case of distortion risk measures, we can provide a sufficient condition for the convexity of $e \mapsto \rho_{1}\left(X_{e}\right)$, which is independent of the distribution of the random variable $Y$. This is the purpose of the next Lemma.

Lemma 3.4. Assume that $\rho_{1}$ is a distortion risk measure (see (2.1)) with a distortion function $\psi$ such that $e \mapsto \psi[p(e)]$ is convex. Then both functions $e \mapsto \rho_{1}\left(X_{e}\right)$ and $e \mapsto \mathbb{E}\left[X_{e}\right]$ are convex.

Proof. To prove the convexity of $e \mapsto \rho_{1}\left(X_{e}\right)$, we write

$$
\rho_{1}\left(X_{e}\right)=\int_{0}^{+\infty} \psi\left(\mathbb{P}\left(X_{e}>x\right)\right) d x=\int_{0}^{+\infty} \psi\left[p(e) P_{Y}((x,+\infty))\right] d x
$$

which is convex, since by the assumption of the Lemma, $e \mapsto \psi\left[p(e) P_{Y}((x,+\infty))\right]$ is convex for any $x \geq 0$. The particular case $\psi(x)=x$ gives that $e \mapsto \mathbb{E}\left[X_{e}\right]$ is convex. 
Remark 3.5. A standard assumption in insurance economics is that $e \mapsto p(e)$ is convex (see [9] for instance), which means that an increased effort leads to a decrease in the loss probability $p(e)$, with a decreasing marginal impact of the effort. In particular, this implies that the objective probabilities $\mathbb{P}\left(X_{e}>x\right)=p(e) P_{Y}((x,+\infty))$ are also decreasing and convex. The assumption in the previous Lemma entails that the subjective probabilities $\psi\left(\mathbb{P}\left(X_{e}>x\right)\right)$ are also decreasing in a convex way. Notice that the assumption that $\psi \circ p$ is convex is also needed in [7]. In Subsection 5.1.2, we provide classes of distortion functions and of maps $e \mapsto p(e)$ satisfying the assumption of the previous Lemma.

We proceed now to solve the problem of IB. By Lemma 3.3, the sets $\mathcal{N}$ and $\mathcal{I}$ take the form

$$
\begin{aligned}
& \mathcal{N}:=\left\{e \in(0,+\infty) \text { such that }(1+\theta)>\frac{\rho_{1}\left(X_{e}\right)}{\mathbb{E}\left[X_{e}\right]}\right\}=\left(0, e_{\theta}\right) \\
& \mathcal{I}:=\left\{e \in(0,+\infty) \text { such that }(1+\theta) \leq \frac{\rho_{1}\left(X_{e}\right)}{\mathbb{E}\left[X_{e}\right]}\right\}=\left(e_{\theta},+\infty\right)
\end{aligned}
$$

where $e_{\theta}:=\inf \left\{e \in \mathbb{R}^{+} \mid \frac{\rho_{1}\left(X_{e}\right)}{\mathbb{E}\left[X_{e}\right]} \geq 1+\theta\right\}$.

We know that for all $e<+\infty, \mathbb{E}\left[X_{e}\right]=p(e) \mathbb{E}[Y] \neq 0$ (since $Y$ is positive a.s.) and from Remarks 2.1 and 2.2 (or from Lemma 3.4), we know that the functions $e \mapsto \rho_{1}\left(X_{e}\right)$ and $e \mapsto \mathbb{E}\left[X_{e}\right]$ are continuous. We have that $e \mapsto \frac{\rho_{1}\left(X_{e}\right)}{\mathbb{E}\left[X_{e}\right]}$ is continuous hence $e_{\theta}$ is the solution to the equation $\frac{\rho_{1}\left(X_{e}\right)}{\mathbb{E}\left[X_{e}\right]}=1+\theta$. So $e_{\theta}$ is defined as the generalized inverse of $e \mapsto \frac{\rho_{1}\left(X_{e}\right)}{\mathbb{E}\left[X_{e}\right]}-1$, which is non-decreasing, therefore $e_{\theta}$ is a non-decreasing function of $\theta$.

We proceed now with a similar analysis to the one developed in Section 3.2, based on finding the minima of the functions $L_{\mathcal{N}}$ and $L_{\mathcal{I}}^{\theta}$ on their respective domains and compare them properly. Recall that $L_{\mathcal{N}}(e)=$ $\rho\left(X_{e}\right)+c(e)$ and $L_{\mathcal{I}}^{\theta}(e)=(1+\theta) \mathbb{E}\left[X_{e}\right]+c(e)$.

\section{On the set $\mathcal{N}$}

Since $L_{\mathcal{N}}$ is convex, non-negative and coercive, it admits an unique minimizer on $\mathbb{R}^{+}$that we denote $e_{N}$. Since the random variables $\left(X_{e}\right)_{e \in \mathbb{R}^{+}}$have finite risk measure, the function $L_{\mathcal{N}}$ is finite at $e=0$, the right hand derivative $\partial_{e}^{+} L_{\mathcal{N}}(0)$ is well defined and we can suppose without loss of generality that it is non-positive.

Proposition 3.4. There exists a positive constant $\theta_{N}$ such that

(a) $\theta \leq \theta_{N} \Longrightarrow \operatorname{argmin} L_{\mathcal{N}}=\left\{e_{\theta}\right\}$,

(b) $\theta>\theta_{N} \Longrightarrow \operatorname{argmin} L_{\mathcal{N}}=\left\{e_{N}\right\}$.

Proof. We define $\theta_{N}:=\inf \left\{\theta \geq 0 \mid e_{\theta}>e_{N}\right\}$. Since $\theta \mapsto e_{\theta}$ is a non-decreasing function, from the definition of $\theta_{N}, \theta>\theta_{N}$ implies that $e_{N} \in \mathcal{N}$ therefore $e_{N}$ is the local minimizer of $L_{\mathcal{N}}$ on $\mathcal{N}$. If $\theta \leq \theta_{N}$ then $L_{\mathcal{N}}$ is decreasing on $\mathcal{N}$ which means that its minimizer is given by the boundary point $e_{\theta}$.

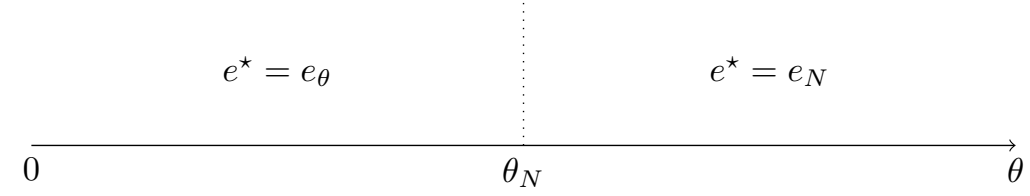

Figure 1: Local minimizer of $L_{\mathcal{N}}$ on the set $\mathcal{N}$, as a function of $\theta$. 


\section{On the set $\mathcal{I}$}

Since $L_{\mathcal{I}}^{\theta}$ is a convex, coercive function it admits an unique minimizer on $\mathbb{R}^{+}$that we call $e_{I}(\theta)$. We will denote it $e_{I}$ when no confusion can arise. The same argument as in the proof of Proposition 3.2 gives that $e_{I}$ is a non-decreasing function of $\theta$. Different from the previous section, in this setting there is no obvious way to compare $e_{I}$ and $e_{\theta}$ with respect to the values of $\theta$ because both efforts are non-decreasing functions of $\theta$.

\section{Global minimum}

We recall that on $\mathcal{N}$ we have $\rho_{1}\left(X_{e}\right)<(1+\theta) \mathbb{E}\left[X_{e}\right]$ and on $\mathcal{I}$ we have the reverse inequality. Therefore we can distinguish six possible cases concerning the order of $e_{I}, e_{N}$ and $e_{\theta}$ depending on the value of $\theta$
(a) $e_{\theta} \leq e_{I} \leq e_{N}$
(c) $e_{N} \leq e_{I} \leq e_{\theta}$
(e) $e_{\theta} \leq e_{N} \leq e_{I}$
(b) $e_{I} \leq e_{\theta} \leq e_{N}$,
(d) $e_{I} \leq e_{N} \leq e_{\theta}$
(f) $e_{N} \leq e_{\theta} \leq e_{I}$

In the next Proposition, we prove that case (b) is not possible and we solve case (a).

Proposition 3.5. If $e_{I} \leq e_{N}$ and $e_{\theta} \leq e_{N}$ then $e_{\theta} \leq e_{I}$ and the global minimum of $L_{1}$ is $e_{I}$.

Proof. Let us prove this Proposition by contradiction. Suppose $e_{I}<e_{\theta}$, since $e_{I}$ is the minimum of the convex function hence $L_{\mathcal{I}}^{\theta}$ is non-decreasing on $\left[e_{\theta},+\infty\right)$ so $\partial_{e}^{+} L_{\mathcal{I}}^{\theta}\left(e_{\theta}\right)>0$. Moreover from the definition of the sets $\mathcal{N}$ and $\mathcal{I}$ we have that $\partial_{e}^{+} L_{\mathcal{N}}\left(e_{\theta}\right)>\partial_{e}^{+} L_{\mathcal{I}}^{\theta}\left(e_{\theta}\right)>0$ and $\partial_{e}^{-} L_{\mathcal{N}}\left(e_{\theta}\right)<\partial_{e}^{-} L_{\mathcal{I}}^{\theta}\left(e_{\theta}\right)$. Since $L_{\mathcal{N}}$ is convex in $e_{N}$ this means that $e_{N}<e_{\theta}$.

We proved that if $e_{I} \leq e_{N}$ and $e_{\theta} \leq e_{N}$ then $e_{\theta} \leq e_{I}$ so $e_{I}$ and $e_{N}$ belong to the set $\mathcal{I}$. Moreover we know for all $e \in \mathcal{I}, L_{\mathcal{I}}^{\theta}(e)<L_{\mathcal{N}}(e)$ therefore $L_{\mathcal{I}}^{\theta}\left(e_{I}\right)<L_{\mathcal{N}}\left(e_{N}\right)$. This means that $e_{I}$ is the global minimum of $L_{1}$.

Remark 3.6. The last argument of this proof let us conclude also for the case (e). Indeed, in this case $e_{N}$ and $e_{I}$ belong to the set $\mathcal{I}$ therefore the same argument applies and $e_{I}$ will be the global minimum of $L_{1}$.

The cases (c) and (d) are adressed in the Proposition that follows.

Proposition 3.6. If $e_{N} \leq e_{\theta}$ and $e_{I} \leq e_{\theta}$ then the global minimum of $L_{1}$ is $e_{N}$.

Proof. If $e_{N} \leq e_{\theta}$ and $e_{I} \leq e_{\theta}$, then $e_{N}$ and $e_{I}$ both belong to the set $\mathcal{N}$. Moreover, for all $e \in \mathcal{N}$ we have $L_{\mathcal{I}}^{\theta}(e)>L_{\mathcal{N}}(e)$, which implies $L_{\mathcal{I}}^{\theta}\left(e_{I}\right)>L_{\mathcal{N}}\left(e_{I}\right) \geq L_{\mathcal{N}}\left(e_{N}\right)$, since $e_{N}$ is the global minimum of $L_{\mathcal{N}}$. So we can conclude that $e_{N}$ is the global minimum of $L_{1}$.

The Theorem that follows addresses the last case (f).

Theorem 3.2. There exists a positive constant $\theta_{M}$ such that

(i) If $e_{N} \leq e_{\theta} \leq e_{I}$ and $\theta \leq \theta_{M}$, the global minimum of $L_{1}$ is $e_{I}$.

(ii) If $e_{N} \leq e_{\theta} \leq e_{I}$ and $\theta>\theta_{M}$, the global minimum of $L_{1}$ is $e_{N}$.

Moreover we have the following inequality

$$
\theta_{N} \leq \theta_{M}
$$

where $\theta_{N}$ is defined in Proposition 3.4. 
Proof. Notice that for all effort $e$, the objective function $L_{\mathcal{I}}^{\theta}$ as a function of $\theta$ is non-decreasing. Indeed,

$$
\theta_{1}<\theta_{2} \Longleftrightarrow\left(1+\theta_{1}\right) \mathbb{E}\left[X_{e}\right]+c(e)<\left(1+\theta_{2}\right) \mathbb{E}\left[X_{e}\right]+c(e) \Longleftrightarrow L_{\mathcal{I}}^{\theta_{1}}(e)<L_{\mathcal{I}}^{\theta_{2}}(e) \forall e .
$$

In particular for $e=e_{I}(\theta), L_{\mathcal{I}}^{\theta}\left(e_{I}(\theta)\right)$ is non-decreasing with $\theta$.

Since $L_{\mathcal{N}}\left(e_{N}\right)$ is independent of $\theta$ and since in the absence of loading factor it is better to have full insurance coverage than no coverage, that is $L_{\mathcal{I}}^{\theta}\left(e_{I}(0)\right)<L_{\mathcal{N}}\left(e_{N}\right)$, we define $\theta_{M}:=\sup \left\{\theta \geq 0 \mid L_{\mathcal{I}}^{\theta}\left(e_{I}(\theta)\right) \leq L_{\mathcal{N}}\left(e_{N}\right)\right\}$. By the definition of $\theta_{M},(i)$ and $(i i)$ hold true.

Let us prove that $\theta_{M} \geq \theta_{N}$ by contradiction. Suppose that $\theta_{N}>\theta_{M}$ and let $\theta$ be such that $\theta_{N}>\theta>\theta_{M}$. By the definition of these two constants we have that $e_{\theta} \leq e_{N}$ and $L_{\mathcal{I}}^{\theta}\left(e_{I}\right)>L_{\mathcal{N}}\left(e_{N}\right)$. This is not possible, since the global optimality of $e_{I}$ and the point $e_{N}$ belonging to $\mathcal{I}$ imply $L_{\mathcal{I}}^{\theta}\left(e_{I}\right) \leq L_{\mathcal{I}}^{\theta}\left(e_{N}\right) \leq L_{\mathcal{N}}\left(e_{N}\right)$.

We can summarize the results of this section by the graphics that follows

$\begin{array}{ccccc}\alpha^{\star}=1 & \vdots & \alpha^{\star}=1 & & \alpha^{\star}=0 \\ e^{\star}=e_{I} & \vdots & e^{\star}=e_{I} & e^{\star}=e_{N} \\ 0 & \theta_{N} & & \theta_{M}\end{array}$

Figure 2: Optimal effort with full insurance coverage depending on the value of $\theta$ : comparisons.

$\underset{0}{\theta_{M}=e_{I}} \quad e^{\star}=e_{N}$

Figure 3: Optimal effort with full insurance coverage depending on the value of $\theta$ : final results.

Let us conclude this Section with the following Corollary stating that statement (B) holds true in the case of self-protection. The behavior of the optimal effort $e^{\star}(\theta)$ is very similar to the one described in [30] and [7].

Corollary 3.2. The map $\theta \mapsto e^{\star}(\theta)$ is non-decreasing for $\theta \leq \theta_{M}$. It becomes constant, equal to $e_{N}$, for $\theta>\theta_{M}$. It has a jump at $\theta=\theta_{M}$ equal to $e_{N}-e_{I}\left(\theta_{M}\right)$. The cases $(c)$, (e) and $(f)$ described above imply that this jump can be negative, from which we deduce that statement (B) holds true.

\section{The insurance seller's optimization problem}

We now move to the problem of IS, which consists into finding the best premium for the insurance coverage that IB is willing to accept. Recall that IS uses a law-invariant and coherent risk measure $\rho_{2}$ and her goal is to solve problem (2.5). Since we are considering safety loading premiums, the problem of IS reduces to

$$
\underset{\theta \in \mathbb{R}_{+}}{\operatorname{minimize}} \alpha^{\star}(\theta)\left(\rho_{2}\left(X_{e^{\star}(\theta)}\right)-(1+\theta) \mathbb{E}\left[X_{e^{\star}(\theta)}\right]\right) .
$$

Remark 4.1. In a standard Principal-Agent relationship, it is assumed that the agent accepts only contracts for which his utility (loss) is greater (less) than some reservation value $R_{0}$, representing the utility (loss) he can guarantee under some outside option. However, this feature is endogenous in our model, since IB is free to choose $\alpha^{\star}=0$ when the contract does not seem convenient for him. 


\subsection{Solving the problem of IS}

In this section we describe the general methodology to solve the problem of IS. This analysis applies to both the self-insurance and self-protection type of IB, described in subsections 3.2 and 3.3.

We know that under Assumption 3.1 or 3.2 , there exists a positive constant $\theta_{M}$ such that $\alpha^{\star}(\theta)=\mathbf{1}_{\theta \in\left[0, \theta_{M}\right]}$. Given the form of the objective function of IS, her problem is reduced to a minimization over a compact set.

$$
\underset{\theta \in\left[0, \theta_{M}\right]}{\operatorname{minimize}} \quad \rho_{2}\left(X_{e_{I}(\theta)}\right)-(1+\theta) \mathbb{E}\left[X_{e_{I}(\theta)}\right]
$$

Therefore, we have by continuity the existence of an optimal loading factor $\theta^{\star}$ which minimizes the risk measure of the loss of IS. Moreover, if the functions involved are smooth enough (which is the case in most of the models studied in the literature), we can characterize $\theta^{\star}$ as the solution to the following system

$$
\begin{aligned}
\left(\frac{\partial}{\partial e} \rho_{2}\left(X_{e_{I}(\theta)}\right)-(1+\theta) \frac{\partial}{\partial e} \mathbb{E}\left[X_{e_{I}(\theta)}\right]\right) \frac{\partial e_{I}(\theta)}{\partial \theta} & =\mathbb{E}\left[X_{e_{I}(\theta)}\right]-\lambda, \\
\lambda\left(\theta-\theta_{M}\right) & =0
\end{aligned}
$$

where $\lambda \geq 0$ is a KKT multiplier associated to the constraint $\theta \leq \theta_{M}$.

Not much more can be said about IS's problem, without specifying a particular model. In Section 5.1, as an application, we solve explicitely the problem of IS in the Pareto case.

\subsection{Adverse Selection}

As an extension of the problem of IS, suppose now a situation in which there are two types of IB in the population. Let us assume directly that the losses have the form (3.3), and the difference between the types of IB is the probability $p$ of having a loss.

More precisely, let $p_{b}>p_{g}$ and let us refer to the two types of IB as the good type and the bad type. Then the distribution of the losses for IB of good and bad type are respectively

$$
\begin{aligned}
& P_{e}^{g}:=\left(1-p_{g}\right) \delta_{\{0\}}+p_{g} P_{Y_{e}}, \\
& P_{e}^{b}:=\left(1-p_{b}\right) \delta_{\{0\}}+p_{b} P_{Y_{e}} .
\end{aligned}
$$

We assume the type of a particular IB is private information. When asking for insurance, IS does not know what is the type of IB but only the distribution of types in the population: with probability $\pi_{g}$, the IB that she is interacting with is of good type and with probability $\pi_{b}$, he is of bad type.

In this setting, we will consider two different problems that may be of interest for IS, the shutdown problem and the screening problem. As explained for instance in [11, 23], the difference between these two problems lies on whether IS wants to sign contracts with the IB of bad type, so they are defined by different optimization programs.

\section{Shutdown problem}

In the shutdown problem, IS wants to provide insurance only to the good type of IB, because of the low risk probability $p_{g}$ of suffering a loss compared to $p_{b}$. In other words, IS wants to exclude the bad type of IB and sign a contract only with IB of good type. IS chooses then a premium that will be rejected by IB of bad type and will only be accepted by IB of good type. 
Given any loading factor $\theta \geq 0$, denote by $\left(\alpha_{g}^{\star}(\theta), e_{g}^{\star}(\theta)\right)$ and $\left(\alpha_{b}^{\star}(\theta), e_{b}^{\star}(\theta)\right)$ the optimal level of insurance and effort chosen by IB of good and bad type respectively. The shutdown problem is given as follows

$$
\begin{array}{ccrl}
\underset{\theta \geq 0}{\operatorname{minimize}} & \rho_{2}\left(X_{e_{g}^{\star}(\theta)}\right)-(1+\theta) \mathbb{E}\left[X_{e_{g}^{\star}(\theta)}\right] \\
\text { s.t. } & \alpha_{b}^{\star}(\theta)=0, \\
& \alpha_{g}^{\star}(\theta)=1 .
\end{array}
$$

In Section 5.1, we approach the shutdown problem of IS in the Pareto case.

\section{Screening problem}

In the screening problem, IS is willing to contract both types of IB and differentiate them by designing simultaneously two premiums, one for each type. Since IB's type is private and he could claim being of any type, IS will design a menu of premiums satisfying the so-called truth-revealing property, that is to say, providing incentives to IB for choosing the contract made for his respective type.

Denote by $L_{1}^{g}$ and $L_{1}^{b}$ respectively the risk measures associated to the total loss of IB of type good and bad. The screening problem faced by IS is given as follows

$$
\begin{aligned}
& \inf _{\theta_{g}, \theta_{b} \geq 0} \pi_{\ell}\left(\rho_{2}\left(X_{e_{g}^{\star}\left(\theta_{g}\right)}\right)-\left(1+\theta_{g}\right) \mathbb{E}\left[X_{e_{g}^{\star}\left(\theta_{g}\right)}\right]\right)+\pi_{h}\left(\rho_{2}\left(X_{e_{b}^{\star}\left(\theta_{b}\right)}\right)-\left(1+\theta_{b}\right) \mathbb{E}\left[X_{e_{b}^{\star}\left(\theta_{b}\right)}\right]\right), \\
& \text { such that }\left\{\begin{array}{l}
L_{1}^{g}\left(\alpha_{g}^{\star}\left(\theta_{g}\right), e_{g}^{\star}\left(\theta_{g}\right)\right) \leq L_{1}^{g}\left(\alpha_{g}^{\star}\left(\theta_{b}\right), e_{g}^{\star}\left(\theta_{b}\right)\right), \\
L_{1}^{b}\left(\alpha_{b}^{\star}\left(\theta_{b}\right), e_{b}^{\star}\left(\theta_{b}\right)\right) \leq L_{1}^{b}\left(\alpha_{b}^{\star}\left(\theta_{g}\right), e_{b}^{\star}\left(\theta_{g}\right)\right),
\end{array}\right. \\
& \text { and such that }\left\{\begin{array}{l}
\alpha_{g}^{\star}\left(\theta_{g}\right)=1, \\
\alpha_{b}^{\star}\left(\theta_{b}\right)=1 .
\end{array}\right.
\end{aligned}
$$

Constraint (4.3) states that whatever the type of IB is, he does not have any incentive to lie and take the premium designed for the other type, since the loss when doing so is greater than the one obtained when revealing the truth. Constraint (4.4) makes sure that the premiums are sufficiently attractive to both types of IB in the population.

In Section 5.1, we solve explicitly the screening problem of IS in the Pareto case.

\section{Case studies}

\subsection{The Pareto Case}

In the following case study, we consider that the distribution of the loss random variable $X_{e}$ is given by

$$
P_{X_{e}}=(1-p) \delta_{\{0\}}+p P_{Y_{e}}
$$

where $0<p<1, \delta_{\{0\}}$ is the Dirac mass at 0 and $P_{Y_{e}}$ denotes the distribution of a Pareto random variable of parameter $\hat{x}>0$ and $k>0$. The Pareto distribution is often used in standard actuarial models (estimation of risk premiums or quotation in non-proportional reinsurance for example).

For tractability purposes, we choose to define

$$
\Pi\left(X_{e}\right)=(1+\theta) \mathbb{E}\left[X_{e}\right], \quad c(e)=\frac{e^{2}}{2}, \quad \psi_{1}(u)=u^{r_{1}}, r_{1} \in(0,1), \quad \psi_{2}(u)=u^{r_{2}}, r_{2} \in(0,1) .
$$




\subsubsection{Self-insurance}

To study self-insurance in this context, we suppose that IB's effort to reduce the size of the loss expresses itself in the control of the parameter $e \mapsto k(e)$. We assume this function to be non-decreasing, concave and such that $k(0)=1$. We then have the following

$$
\mathbb{P}\left[X_{e}>x\right]=p\left(\frac{\hat{x}}{x}\right)^{k(e)}, \forall x>\hat{x}, \quad \mathbb{E}\left[X_{e}\right]=\frac{p \hat{x} k(e)}{k(e)-1}, \quad \bar{q}_{X_{e}}(u)= \begin{cases}\hat{x}\left(\frac{p}{u}\right)^{1 / k(e)}, & \text { if } u \in[0, p], \\ 0, & \text { if } p<u \leq 1 .\end{cases}
$$

Notice that since $k$ is a concave function, the quantile function $\bar{q}_{X_{e}}$ is convex. We can calculate the risk measure $\rho_{1}\left(X_{e}\right)$ which is given by Equation (2.1)

$$
\rho_{1}\left(X_{e}\right)=r_{1} \hat{x} \int_{0}^{p} u^{r_{1}-1-1 / k(e)} p^{1 / k(e)} d u= \begin{cases}\frac{r_{1} p^{r_{1}} \hat{x} k(e)}{r_{1} k(e)-1}, & \text { if } k(e)>\frac{1}{r_{1}}, \\ +\infty, & \text { otherwise. }\end{cases}
$$

Therefore, IB's problem is

$$
\inf _{(\alpha, e) \in[0,1] \times(0, \infty)}\left\{(1-\alpha) \frac{p^{r_{1}} r_{1} \hat{x} k(e)}{r_{1} k(e)-1}+\alpha(1+\theta) \frac{p \hat{x} k(e)}{k(e)-1}+\frac{e^{2}}{2}\right\} .
$$

Remark 5.1. The explicit value of $\rho_{1}\left(X_{e}\right)$ given above entails that IB will never choose an effort below the level $k^{-1}\left(\frac{1}{r_{1}}\right)$. This also implies that $k(e)>\frac{1}{r_{1}}>1$.

Moreover we can compute the value of the function $G$ defined by Equation (3.2)

$$
G(e)=\frac{\rho_{1}\left(X_{e}\right)}{\mathbb{E}\left[X_{e}\right]}=\frac{r_{1} p^{r_{1}-1}(k(e)-1)}{r_{1} k(e)-1},
$$

so $G$ is decreasing and we can apply all the results of Subsection 3.2 to this case.

To solve problem (5.1), we start by characterizing the main constants introduced in Section 3 . The point $e_{\theta}$ is given by

$$
e_{\theta}=k^{-1}\left(\frac{1+\theta-p^{r_{1}-1} r_{1}}{r_{1}\left(1+\theta-p^{r_{1}-1}\right)}\right)
$$

and the corresponding sets $\mathcal{N}$ and $\mathcal{I}$

$$
\begin{aligned}
& \mathcal{N}=\left\{e \in \mathbb{R}^{+} \mid(1+\theta)>\frac{p^{r_{1}-1} r_{1}(k(e)-1)}{\left(r_{1} k(e)-1\right)}\right\}=\left(e_{\theta},+\infty\right), \\
& \mathcal{I}=\left\{e \in \mathbb{R}^{+} \mid(1+\theta) \leq \frac{p^{r_{1}-1} r_{1}(k(e)-1)}{\left(r_{1} k(e)-1\right)}\right\}=\left[0, e_{\theta}\right] .
\end{aligned}
$$

As the global minimum of $L_{\mathcal{N}}$, the point $e_{N}$ is characterized by the optimality condition

$$
e_{N}=\frac{p^{r_{1}} r_{1} \hat{x} k^{\prime}\left(e_{N}\right)}{\left(r_{1} k\left(e_{N}\right)-1\right)^{2}}
$$

which shows that $e_{N}$ is a non-decreasing function of the risk probability $p$.

As the global minimum of $L_{\mathcal{I}}^{\theta}, e_{I}(\theta)$ is characterized by the equation $L_{\mathcal{I}}^{\prime}\left(e_{I}(\theta)\right)=0$ which rewrites as

$$
e_{I}(\theta)=\frac{(1+\theta) \hat{x} p k^{\prime}\left(e_{I}(\theta)\right)}{\left(k\left(e_{I}(\theta)\right)-1\right)^{2}} .
$$

We observe then that $e_{I}$ is non-decreasing with $p$ and with $\theta$.

Finally, we have from Theorem 3.1 the existence of a constant $\theta_{M}$ that defines the global minimum of the objective function $L_{1}$. The point $\theta_{M}$ is the solution to the equation $L_{\mathcal{I}}^{\theta_{M}}\left(e_{I}\left(\theta_{M}\right)\right)=L_{\mathcal{N}}\left(e_{N}\right)$ and we have 
- If $\theta \leq \theta_{M}$ then the solution to $(5.1)$ is $\left(\alpha^{\star}, e^{\star}\right)=\left(1, e_{I}(\theta)\right)$.

- If $\theta>\theta_{M}$ then the solution to $(5.1)$ is $\left(\alpha^{\star}, e^{\star}\right)=\left(0, e_{N}\right)$.

Concerning the problem of IS, we can calculate the distortion risk measure $\rho_{2}\left(X_{e}\right)$

$$
\rho_{2}\left(X_{e}\right)=r_{2} \hat{x} \int_{0}^{p} u^{r_{2}-1-1 / k(e)} p^{1 / k(e)} d u= \begin{cases}\frac{p^{r_{2}} r_{2} \hat{x} k(e)}{r_{2} k(e)-1}, & \text { if } k(e)>\frac{1}{r_{2}}, \\ +\infty, & \text { otherwise. }\end{cases}
$$

Therefore, problem (4.1) writes

$$
\inf _{\theta \in \Theta_{M}}\left\{\frac{p^{r_{2}} r_{2} \hat{x} k\left(e_{I}(\theta)\right)}{r_{2} k\left(e_{I}(\theta)\right)-1}-(1+\theta) \frac{p \hat{x} k\left(e_{I}(\theta)\right)}{k\left(e_{I}(\theta)\right)-1}\right\}
$$

where $\Theta_{M}:=\left\{\theta \in\left[0, \theta_{M}\right]: k\left(e_{I}(\theta)\right)>\frac{1}{r_{2}}\right\}$. In the Pareto case, IS's problem is easier to solve than in the general case, because its objective function turns out to be monotone. In fact, we provide now the solution to the problem of IS, which is given by the maximum premium IB is willing to pay.

Theorem 5.1. The solution to problem (5.4) is $\theta^{\star}=\theta_{M}$.

Proof. We prove that the objective function of IS is non-increasing, from which the conclusion of the Lemma follows. Write it as the sum of two functions of $\theta$ for which we study the monotonicity:

$$
L_{2}(\theta):=f_{1}(\theta)-f_{2}(\theta)
$$

with

$$
f_{1}:=\frac{p^{r_{2}} r_{2} \hat{x} k\left(e_{I}(\theta)\right)}{r_{2} k\left(e_{I}(\theta)\right)-1}, \quad f_{2}:=(1+\theta) \frac{p \hat{x} k\left(e_{I}(\theta)\right)}{k\left(e_{I}(\theta)\right)-1} .
$$

Recall that $e_{I}(\theta)$ is increasing in $\theta$ and then so is $k\left(e_{I}(\theta)\right)$. Notice that $f_{1}$ is a non-increasing function as

$$
\frac{\partial f_{1}}{\partial \theta}=-\frac{p^{r_{2}} \hat{x} r_{2} k^{\prime}\left(e_{I}(\theta)\right) e_{I}^{\prime}(\theta)}{\left(r_{2} k\left(e_{I}(\theta)\right)-1\right)^{2}}<0 .
$$

We now study the derivative of $f_{2}$. Rewrite the characterization of $e_{I}(\theta)$ as follows

$$
\frac{e_{I}(\theta)\left(k\left(e_{I}(\theta)\right)-1\right)^{2}}{p \hat{x} k^{\prime}\left(e_{I}(\theta)\right)}-(1+\theta)=0 .
$$

Consider the following function

$$
F(\theta, e):=\frac{e(k(e)-1)^{2}}{p \hat{x} k^{\prime}(e)}-(1+\theta) .
$$

By the Implicit Function Theorem, we have

$$
\frac{\partial e_{I}(\theta)}{\partial \theta}=\frac{-\partial F\left(\theta, e_{I}(\theta)\right) / \partial \theta}{\partial F\left(\theta, e_{I}(\theta)\right) / \partial e}
$$

Remark that

$$
\begin{aligned}
\frac{\partial F(\theta, e)}{\partial e} & =\frac{\left((k(e)-1)^{2}+2 e k^{\prime}(e)(k(e)-1)\right) k^{\prime}(e) p \hat{x}-p \hat{x} k^{\prime \prime}(e) e(k(e)-1)^{2}}{\left(p \hat{x} k^{\prime}(e)\right)^{2}} \\
& =\frac{(k(e)-1)^{2}}{p \hat{x} k^{\prime}(e)}+\frac{2 e k^{\prime}(e)(k(e)-1)}{p \hat{x} k^{\prime}(e)}-\frac{k^{\prime \prime}(e) e}{k^{\prime}(e)} \frac{(k(e)-1)^{2}}{p \hat{x} k^{\prime}(e)}
\end{aligned}
$$


In the next few lines, we will write $e_{I}$ instead of $e_{I}(\theta)$ for simplicity of notation. By rewriting Equation (5.6), we notice that

$$
\frac{\left(k\left(e_{I}\right)-1\right)^{2}}{p \hat{x} k^{\prime}\left(e_{I}\right)}=\frac{(1+\theta)}{e_{I}},
$$

therefore

$$
\frac{\partial F\left(\theta, e_{I}\right)}{\partial e}=\frac{1+\theta}{e_{I}}\left(1+\frac{2 e_{I} k^{\prime}\left(e_{I}\right)}{k\left(e_{I}\right)-1}-\frac{k^{\prime \prime}\left(e_{I}\right) e_{I}}{k^{\prime}\left(e_{I}\right)}\right)
$$

and

$$
\frac{\partial F\left(\theta, e_{I}\right)}{\partial \theta}=-1
$$

This leads to

$$
\frac{\partial e_{I}}{\partial \theta}=\frac{e_{I}}{1+\theta}\left(\frac{1}{1+\frac{2 e_{I} k^{\prime}\left(e_{I}\right)}{k\left(e_{I}\right)-1}-\frac{k^{\prime \prime}\left(e_{I}\right) e_{I}}{k^{\prime}\left(e_{I}\right)}}\right)
$$

Since

$$
1+\frac{2 e_{I} k^{\prime}\left(e_{I}\right)}{k\left(e_{I}\right)-1}-\frac{k^{\prime \prime}\left(e_{I}\right) e_{I}}{k^{\prime}\left(e_{I}\right)}>1, \quad \frac{e_{I}}{1+\theta}>0
$$

we obtain the following inequality

$$
\frac{\partial e_{I}}{\partial \theta}<\frac{e_{I}}{1+\theta}
$$

By differentiating $f_{2}$ and from inequality (5.7) we have

$$
\begin{aligned}
\frac{\partial f_{2}}{\partial \theta} & =p \hat{x} \frac{\left(k\left(e_{I}(\theta)\right)-1\right) k\left(e_{I}(\theta)\right)-(1+\theta) k^{\prime}\left(e_{I}(\theta)\right) e_{I}^{\prime}(\theta)}{\left(k\left(e_{I}(\theta)\right)-1\right)^{2}} \\
& >\frac{p \hat{x}}{\left(k\left(e_{I}(\theta)\right)-1\right)^{2}}\left(\left(k\left(e_{I}(\theta)\right)-1\right) k\left(e_{I}(\theta)\right)-k^{\prime}\left(e_{I}(\theta)\right) e_{I}(\theta)\right) .
\end{aligned}
$$

To conclude, define the following function

$$
h(x):=(k(x)-1) k(x)-x k^{\prime}(x),
$$

its derivative $h^{\prime}(x)=2 k^{\prime}(x)[k(x)-1]-x k^{\prime \prime}(x)$ is positive since $k$ is non-decreasing, concave and bounded from below by 1 (see Remark 5.1). This entails that $h(x)>h(0)=0$, for any $x>0$. Therefore from Inequality (5.8), $f_{2}$ is non-decreasing in $\theta$.

From Equation (5.5), this leads to $L_{2}$ being a non-increasing function of $\theta$. In conclusion the optimal insurance price is the upper bound of the interval where we minimize this function, that is to say $\theta^{\star}=\theta_{M}$.

\section{Adverse selection}

Recall from Subsection 4.2, the shutdown problem of IS

$$
\begin{array}{cc}
\underset{\theta \geq 0}{\operatorname{minimize}} & \rho_{2}\left(X_{e_{g}^{\star}(\theta)}\right)-(1+\theta) \mathbb{E}\left[X_{e_{g}^{\star}(\theta)}\right] \\
\text { s.t. } & \alpha_{b}^{\star}(\theta)=0, \\
& \alpha_{g}^{\star}(\theta)=1 .
\end{array}
$$


IS needs to optimize over the prices $\theta$ such that $\theta \leq \theta_{M}\left(p_{g}\right)$ and $\theta>\theta_{M}\left(p_{b}\right)$ where $\theta_{M}\left(p_{g}\right)$ is the optimal insurance price for the IB of good type and $\theta_{M}\left(p_{b}\right)$ is the optimal insurance price for IB of bad type.

Recall that $\theta_{M}$ is characterized by $L_{\mathcal{I}}^{\theta_{M}}\left(e_{I}\left(\theta_{M}\right)\right)=L_{\mathcal{N}}\left(e_{N}\right)$. From the dependances of $e_{I}(\theta)$ and $e_{N}$ on $p$, we can notice that the higher the probability of risk is for an IB the more he is willing to pay to insure this risk, this leads to $\theta_{M}\left(p_{g}\right) \leq \theta_{M}\left(p_{b}\right)$. Therefore IS cannot choose a price $\theta$ respecting the constraints of the shut-down contract which means that such contract does not exist in our context.

Recall also the screening problem of IS

$$
\begin{aligned}
& \inf _{\theta_{g}, \theta_{b} \geq 0} \pi_{\ell}\left(\rho_{2}\left(X_{e_{g}^{\star}\left(\theta_{g}\right)}\right)-\left(1+\theta_{g}\right) \mathbb{E}\left[X_{e_{g}^{\star}\left(\theta_{g}\right)}\right]\right)+\pi_{h}\left(\rho_{2}\left(X_{e_{b}^{\star}\left(\theta_{b}\right)}\right)-\left(1+\theta_{b}\right) \mathbb{E}\left[X_{e_{b}^{\star}\left(\theta_{b}\right)}\right]\right), \\
& \text { such that }\left\{\begin{array}{l}
L_{1}^{g}\left(\alpha_{g}^{\star}\left(\theta_{g}\right), e_{g}^{\star}\left(\theta_{g}\right)\right) \leq L_{1}^{g}\left(\alpha_{g}^{\star}\left(\theta_{b}\right), e_{g}^{\star}\left(\theta_{b}\right)\right), \\
L_{1}^{b}\left(\alpha_{b}^{\star}\left(\theta_{b}\right), e_{b}^{\star}\left(\theta_{b}\right)\right) \leq L_{1}^{b}\left(\alpha_{b}^{\star}\left(\theta_{g}\right), e_{b}^{\star}\left(\theta_{g}\right)\right),
\end{array}\right. \\
& \text { and such that }\left\{\begin{array}{l}
\alpha_{g}^{\star}\left(\theta_{g}\right)=1, \\
\alpha_{b}^{\star}\left(\theta_{b}\right)=1 .
\end{array}\right.
\end{aligned}
$$

From Equation (5.11), IS chooses a price $\theta_{g}$ such that $\theta_{g} \leq \theta_{M}\left(p_{g}\right)$ and a price $\theta_{b}$ such that $\theta_{b} \leq \theta_{M}\left(p_{b}\right)$. From Equation (5.10), IS needs to select the prices such that $\theta_{g} \leq \theta_{b}$ and $\theta_{b} \leq \theta_{g}$. Therefore the only way in which IS can contract both types of IB is by offering the same price to them $\theta_{g}=\theta_{b}$.

One can observe that adverse selection problems in our context of linear contracts, and coherent risk measures are rather degenerate. The insurance seller cannot use the standard tools to deal with adverse selection: she cannot offer a menu of contracts since the optimal insurance coverage is either 0 or 1 , she cannot exclude the bad type of agent since shut-down contracts do not exist, and she cannot use differentiated pricing since the constraints in the screening problem imply that the price is unique.

\subsubsection{Self-protection}

To study self-protection, we suppose that IB's effort reduces the probability of loss $e \mapsto p(e)$ which we assume non-increasing and convex. We then have the following

$$
\mathbb{P}\left[X_{e}>x\right]=p(e)\left(\frac{\hat{x}}{x}\right)^{k}, \forall x>\hat{x}, \quad \mathbb{E}\left[X_{e}\right]=\frac{\hat{x} k}{k-1} p(e), \quad \bar{q}_{X_{e}}(u)= \begin{cases}\hat{x}\left(\frac{p(e)}{u}\right)^{1 / k}, & \text { if } u \in[0, p(e)], \\ 0, & \text { if } p(e)<u \leq 1 .\end{cases}
$$

We can notice that since $p$ is a convex function, the quantile function $\bar{q}_{X_{e}}$ is also a convex function of $e$. From Equation (2.1) the risk measure $\rho_{1}\left(X_{e}\right)$ is

$$
\rho_{1}\left(X_{e}\right)=r_{1} \hat{x} \int_{0}^{p(e)} u^{r_{1}-1-1 / k} p^{1 / k} d u= \begin{cases}\frac{r_{1} p(e)^{r_{1}} \hat{x} k}{r_{1} k-1}, & \text { if } k>\frac{1}{r_{1}} \\ +\infty, & \text { otherwise }\end{cases}
$$

Therefore, IB's problem is

$$
\inf _{(\alpha, e) \in[0,1] \times(0, \infty)}\left\{(1-\alpha) \frac{r_{1} \hat{x} k}{r_{1} k-1} p(e)^{r_{1}}+\alpha(1+\theta) \frac{\hat{x} k}{k-1} p(e)+\frac{e^{2}}{2}\right\} .
$$

We can also compute the value of the function $G$ from Equation (3.2)

$$
G(e)=\frac{\rho_{1}\left(X_{e}\right)}{\mathbb{E}\left[X_{e}\right]}=\frac{r_{1}(k-1)}{r_{1} k-1} p(e)^{r_{1}-1},
$$


which is a increasing function of $e$ since $p$ is non-increasing and $r_{1}<1$. Therefore we can apply the results of Subsection 3.3. In this setting, $e_{\theta}$ is defined by

$$
e_{\theta}=p^{-1}\left(\left(\frac{(1+\theta)\left(r_{1} k-1\right)}{r_{1}(k-1)}\right)^{1 /\left(r_{1}-1\right)}\right),
$$

which is an increasing function of $\theta$ and the sets $\mathcal{N}$ and $\mathcal{I}$ are defined as follows

$$
\begin{aligned}
& \mathcal{N}=\left\{e \in(0,+\infty) \text { such that }(1+\theta)>\frac{\rho_{1}\left(X_{e}\right)}{\mathbb{E}\left[X_{e}\right]}\right\}=\left(0, e_{\theta}\right), \\
& \mathcal{I}=\left\{e \in(0,+\infty) \text { such that }(1+\theta)<\frac{\rho_{1}\left(X_{e}\right)}{\mathbb{E}\left[X_{e}\right]}\right\}=\left(e_{\theta},+\infty\right) .
\end{aligned}
$$

We have

$$
L_{\mathcal{N}}(e)=\frac{r_{1} \hat{x} k}{r_{1} k-1} p(e)^{r_{1}}+\frac{e^{2}}{2}, \quad L_{\mathcal{I}}^{\theta}(e)=\frac{(1+\theta) \hat{x} k}{k-1} p(e)+\frac{e^{2}}{2}
$$

If the map $u \mapsto \bar{q}_{Y}(u)$ is convex and if $\frac{2}{p} \geq \frac{p^{\prime \prime}}{\left(p^{\prime}\right)^{2}}$, then Assumption 2.1 is satisfied, and by Theorem 2.1, $L_{\mathcal{N}}$ is a convex function of $e$. Alternatively, since the expression for $L_{\mathcal{N}}$ is more explicit here, we can give another condition: $L_{\mathcal{N}}$ is convex if and only if $e \mapsto p(e)^{r_{1}}$ is convex, which is the case if $\frac{p^{\prime \prime}}{\left(p^{\prime}\right)^{2}} \geq \frac{1-r_{1}}{p}$. An example of function satisfying this last condition is given by $p(e)=\frac{1}{a+e}$, with $a>1$.

Let us now study the minimum of the function $L_{\mathcal{N}}$. Its first derivative is given by:

$$
L_{\mathcal{N}}^{\prime}(e)=e+\frac{r_{1}^{2} \hat{x} k}{r_{1} k-1} p^{\prime}(e) p(e)^{r_{1}-1}
$$

Since

$$
L_{\mathcal{N}}^{\prime}(0)=\frac{r_{1}^{2} \hat{x} k}{r_{1} k-1} p^{\prime}(0) p(0)^{r_{1}-1}<0, \text { and } \lim _{e \rightarrow+\infty} L_{\mathcal{N}}^{\prime}(e)=+\infty,
$$

the global minimum $e_{N}$ of the function $L_{\mathcal{N}}$ is thus the solution to

$$
e_{N}=-\frac{r_{1}^{2} \hat{x} k}{r_{1} k-1} p^{\prime}\left(e_{N}\right) p\left(e_{N}\right)^{r_{1}-1}
$$

The global minimum of $L_{\mathcal{I}}$ is characterized by

$$
e_{I}(\theta)=-\frac{(1+\theta) \hat{x} k}{k-1} p^{\prime}\left(e_{I}(\theta)\right)
$$

This equation allows us to conclude that $e_{I}(\theta)$ is an increasing function of $\theta$. We have from Theorem 3.2 the existence of a constant $\theta_{M}$ that defines the global minimum of the objective function $L_{1}$. The point $\theta_{M}$ is the solution to the equation $L_{\mathcal{I}}^{\theta_{M}}\left(e_{I}\left(\theta_{M}\right)\right)=L_{\mathcal{N}}\left(e_{N}\right)$ and we have

- If $\theta<\theta_{M}$ then the solution to (5.12) is $\left(\alpha^{\star}, e^{\star}\right)=\left(1, e_{I}(\theta)\right)$.

- If $\theta \geq \theta_{M}$ then the solution to $(5.12)$ is $\left(\alpha^{\star}, e^{\star}\right)=\left(0, e_{N}\right)$.

To study IS's problem in the self-protection case, we will make the following assumption:

\section{Assumption 5.1.}

$$
p(e)=\frac{1}{a+e}, \quad a>1 .
$$


The distortion risk measure is given by

$$
\rho_{2}\left(X_{e}\right)=r_{2} \hat{x} \int_{0}^{p(e)} u^{r_{2}-1-1 / k} p(e)^{1 / k} d u= \begin{cases}\frac{p(e)^{r_{2} r_{2} \hat{x} k}}{r_{2} k-1}, & \text { if } k>\frac{1}{r_{2}} \\ +\infty, & \text { otherwise }\end{cases}
$$

From Section 3 we know that the equilibrium for IB's problem in the self-protection case is $\left(\alpha^{\star}, e^{\star}\right)=\left(1, e_{I}(\theta)\right)$, therefore, IS's problem is as follows

$$
\inf _{\theta \in\left[0, \theta_{M}\right]}\left\{\frac{r_{2} \hat{x} k p\left(e_{I}(\theta)\right)^{r_{2}}}{r_{2} k-1}-(1+\theta) \frac{\hat{x} k p\left(e_{I}(\theta)\right)}{k-1}\right\} .
$$

Given Assumption 5.1, the characterization (5.14) of $e_{I}(\theta)$ now writes

$$
e_{I}(\theta)=c \frac{1+\theta}{\left(a+e_{I}(\theta)\right)^{2}}
$$

with $c:=\hat{x} \frac{k}{k-1}>0$. After some cumbersome, but easy calculations (see Appendix B), we get:

$$
e_{I}(\theta)=t_{1}(\theta)+t_{2}(\theta)-\frac{2 a}{3}
$$

where

$$
\begin{aligned}
& t_{1}(\theta):=\left[\frac{1}{2}\left(c(1+\theta)+\frac{2}{27} a^{3}-\sqrt{\frac{4}{27} c(1+\theta) a^{3}+c^{2}(1+\theta)^{2}}\right)\right]^{1 / 3} \text { and } \\
& t_{2}(\theta):=\left[\frac{1}{2}\left(c(1+\theta)+\frac{2}{27} a^{3}+\sqrt{\frac{4}{27} c(1+\theta) a^{3}+c^{2}(1+\theta)^{2}}\right)\right]^{1 / 3}
\end{aligned}
$$

In this setting of self-protection, the solution to the problem of IS is also to charge the maximum premium $\theta_{M}$ that IB is willing to pay. We have then the following result, whose proof is postponed to Appendix C.

Theorem 5.2. Under Assumption 5.1, the solution to problem (5.15) is $\theta^{\star}=\theta_{M}$.

\subsection{Discrete distributions}

As already mentioned in the Introduction, most of the literature on self-insurance and self-protection concentrates on the case of discrete distributions, with only two possible loss values. We show in this subsection that our results also apply to the case of discrete distributions, with an arbitrary number of possible losses. We prove that, contrary to the Bernoulli case, more conditions are needed for statements (A) and (B) to hold true. When these conditions are not satisfied, this gives simple counter-examples to the standard results obtained in the Bernoulli case.

Let $\left\{p_{i}, i=0, \ldots, n-1\right\}$ be positive numbers such that $\sum_{i=0}^{n-1} p_{i}=1$, and let $0<x_{1}<\cdots<x_{n-1}$ be positive numbers. We consider a discrete loss with $n \in \mathbb{N}$ values, given by

$$
X:= \begin{cases}x_{i} & \text { with probability } p_{i}, i \in[1, n-1] \\ 0 & \text { with probability } p_{0}\end{cases}
$$

To simplify the calculations, we suppose here that the risk measure $\rho_{1}$ is a distortion risk measure, whose definition is given in Section 2, Equation (2.1)

$$
\rho_{1}(X):=\int_{0}^{1} \bar{q}_{X}(u) d \psi(u)=\int_{0}^{+\infty} \psi(\mathbb{P}[X>u]) d u .
$$




\section{Self-insurance: the discrete case.}

To study Self-Insurance in this case, we now suppose that the agent exerts an effort $e$, that has an impact on every possible loss value $x_{i}$, which will be denoted $x_{i}(e)$ and the loss $X$ will be denoted $X_{e}$. Then we can write

$$
\begin{aligned}
\rho_{1}\left(X_{e}\right) & =\int_{0}^{x_{1}(e)} \psi\left(\sum_{i=1}^{n-1} p_{i}\right) d u+\int_{x_{1}(e)}^{x_{2}(e)} \psi\left(\sum_{i=2}^{n-1} p_{i}\right) d u+\ldots+\int_{x_{n-2}(e)}^{x_{n-1}(e)} \psi\left(p_{n-1}\right) d u \\
& =\left(\psi\left(\sum_{i=1}^{n-1} p_{i}\right)-\psi\left(\sum_{i=2}^{n-1} p_{i}\right)\right) x_{1}(e)+\ldots+\psi\left(p_{n-1}\right) x_{n-1}(e), \\
& =\sum_{j=1}^{n-1}\left(\psi\left(\sum_{i=j}^{n-1} p_{i}\right)-\psi\left(\sum_{i=j+1}^{n-1} p_{i}\right)\right) x_{j}(e), \quad \text { with } p_{n}:=0 .
\end{aligned}
$$

To simplify the formulas, we will denote

$$
\kappa_{j}:=\psi\left(\sum_{k=j}^{n-1} p_{k}\right)-\psi\left(\sum_{k=j+1}^{n-1} p_{k}\right) \geq 0, \quad \forall j=1, \ldots, n .
$$

Moreover we have

$$
\mathbb{E}\left[X_{e}\right]=\sum_{j=1}^{n-1} p_{j} x_{j}(e) .
$$

Hence the function $G$ defined in Lemma 3.1 is given by

$$
G(e)=\frac{\rho_{1}\left(X_{e}\right)}{\mathbb{E}\left[X_{e}\right]}=\frac{\sum_{j=1}^{n-1} \kappa_{j} x_{j}(e)}{\sum_{j=1}^{n-1} p_{j} x_{j}(e)} .
$$

Assumption 5.2. We suppose that for all $j$ and for all $i>j$, the function $e \mapsto \frac{x_{j}(e)}{x_{i}(e)}$ is a non-decreasing function (recall that $x_{j}(e)<x_{i}(e)$ ).

This assumption means that the marginal impact of the effort is higher on small losses than it is on catastrophic losses.

We need to study the monotony of $G$ to determine the optimal prevention effort of self-insurance.

$$
\begin{aligned}
G^{\prime}(e) & =\frac{1}{\left(\sum_{j=1}^{n-1} p_{j} x_{j}(e)\right)^{2}}\left(\sum_{j=1}^{n-1} \kappa_{j} x_{j}^{\prime}(e) \sum_{j=1}^{n-1} p_{j} x_{j}(e)-\sum_{j=1}^{n-1} \kappa_{j} x_{j}(e) \sum_{j=1}^{n-1} p_{j} x_{j}^{\prime}(e)\right) \\
& =\frac{1}{\left(\sum_{j=1}^{n-1} p_{j} x_{j}(e)\right)^{2}} \sum_{i, j=1}^{n-1} \kappa_{j} p_{i}\left(x_{i}(e) x_{j}^{\prime}(e)-x_{i}^{\prime}(e) x_{j}(e)\right) \\
& =\frac{1}{\left(\sum_{j=1}^{n-1} p_{j} x_{j}(e)\right)^{2}} \sum_{i \neq j} \kappa_{j} p_{i} a_{i, j}
\end{aligned}
$$


where $a_{j, i}:=x_{i}(e) x_{j}^{\prime}(e)-x_{i}^{\prime}(e) x_{j}(e)$, which implies that $a_{j, i}=-a_{i, j}$. We have that

$$
G^{\prime}(e)=\frac{1}{\left(\sum_{j=1}^{n-1} p_{j} x_{j}(e)\right)^{2}}\left(\sum_{i>j} \kappa_{j} p_{i} a_{i, j}-\sum_{i<j} \kappa_{j} p_{i} a_{j, i}\right)=\frac{1}{\left(\sum_{j=1}^{n-1} p_{j} x_{j}(e)\right)^{2}} \sum_{i>j}\left(\kappa_{j} p_{i}-\kappa_{i} p_{j}\right) a_{i, j} .
$$

Thus, the sign of the derivative $G^{\prime}(e)$ depends on the sign of $\kappa_{j} p_{i}-\kappa_{i} p_{j}$ for all $i>j$. Let us prove that for a concave distortion function, for all $i>j$, we have the inequality $\kappa_{j} p_{i}-\kappa_{i} p_{j} \leq 0$. First we recall the following standard result.

Lemma 5.1. If $f$ is a concave function on an interval $I$, for all points $x, y, z$ in $I$ such that $x<y<z$ we have

$$
\frac{f(z)-f(y)}{z-y} \leq \frac{f(z)-f(x)}{z-x} \leq \frac{f(y)-f(x)}{y-x} .
$$

Proposition 5.1. For all $i>j, \kappa_{j} p_{i}-\kappa_{i} p_{j} \leq 0$.

Proof. Proving that $\kappa_{j} p_{i}-\kappa_{i} p_{j} \leq 0$ is equivalent to proving that $\frac{\kappa_{j}}{p_{j}}-\frac{\kappa_{i}}{p_{i}} \leq 0$.

From Equation (5.21) for all $i>j$

$$
\frac{\kappa_{j}}{p_{j}}-\frac{\kappa_{i}}{p_{i}}=\frac{1}{p_{j}}\left(\psi\left(\sum_{k=j}^{n-1} p_{k}\right)-\psi\left(\sum_{k=j+1}^{n-1} p_{k}\right)\right)-\frac{1}{p_{i}}\left(\psi\left(\sum_{k=i+1}^{n-1} p_{k}\right)-\psi\left(\sum_{k=i}^{n-1} p_{k}\right)\right) .
$$

Applying Lemma 5.1 to $\psi$ and with

$$
x:=\sum_{k=i+1}^{n-1} p_{k}, y:=\sum_{k=j+1}^{n-1} p_{k} \text { and } z:=\sum_{k=j}^{n-1} p_{k},
$$

we obtain

$$
\frac{\kappa_{j}}{p_{j}}-\frac{\kappa_{i}}{p_{i}} \leq \frac{1}{\sum_{k=j}^{i} p_{k}}\left(\psi\left(\sum_{k=j}^{n-1} p_{k}\right)-\psi\left(\sum_{k=i+1}^{n-1} p_{k}\right)\right)-\frac{1}{p_{i}}\left(\psi\left(\sum_{k=i+1}^{n-1} p_{k}\right)-\psi\left(\sum_{k=i}^{n-1} p_{k}\right)\right) .
$$

Applying again Lemma 5.1 to $\psi$ and with

$$
x:=\sum_{k=i+1}^{n-1} p_{k}, y:=\sum_{k=i}^{n-1} p_{k} \text { and } z:=\sum_{k=j}^{n-1} p_{k},
$$

we get

$$
\frac{\kappa_{j}}{p_{j}}-\frac{\kappa_{i}}{p_{i}} \leq \frac{1}{p_{i}}\left(\psi\left(\sum_{k=i}^{n-1} p_{k}\right)-\psi\left(\sum_{k=i+1}^{n-1} p_{k}\right)\right)-\frac{1}{p_{i}}\left(\psi\left(\sum_{k=i+1}^{n-1} p_{k}\right)-\psi\left(\sum_{k=i}^{n-1} p_{k}\right)\right)=0
$$

which ends the proof.

In conclusion, from Assumption 5.2 and Proposition 5.1 we can conclude that $G^{\prime}(e) \leq 0$, therefore $G$ is a non-increasing function of $e$ and statement (A) holds true. 


\section{Self-protection: the discrete case.}

Now, let us focus on the study of Self-Protection: we assume here that the effort only impacts the loss probabilities, which we denote $p_{i}(e)$ for all $i=0, \ldots, n-1$. Using the same calculations as above, we get

$$
G(e):=\frac{\rho_{1}\left(X_{e}\right)}{\mathbb{E}\left[X_{e}\right]}=\frac{\sum_{j=1}^{n-1} \kappa_{j}(e) x_{j}}{\sum_{j=1}^{n-1} p_{j}(e) x_{j}},
$$

with

$$
\kappa_{j}(e):=\psi\left(\sum_{k=j}^{n-1} p_{k}(e)\right)-\psi\left(\sum_{k=j+1}^{n-1} p_{k}(e)\right) \geq 0, \quad \forall j=1, \ldots, n .
$$

As usual, we further assume that for each $i=0, \ldots, n-1, p_{i}$ is a decreasing and convex function of $e$. The first derivative of $G$ writes

$$
\begin{aligned}
G^{\prime}(e) & =\frac{1}{\left(\sum_{j=1}^{n-1} p_{j}(e) x_{j}\right)^{2}}\left(\sum_{j=1}^{n-1} \kappa_{j}^{\prime}(e) x_{j} \sum_{i=1}^{n-1} p_{i}(e) x_{i}-\sum_{j=1}^{n-1} \kappa_{j}(e) x_{j} \sum_{i=1}^{n-1} p_{i}^{\prime}(e) x_{i}\right), \\
& =\frac{1}{\left(\sum_{j=1}^{n-1} p_{j}(e) x_{j}\right)^{2}} \sum_{i, j=1}^{n-1} x_{j} x_{i}\left(\kappa_{j}^{\prime}(e) p_{i}(e)-p_{i}^{\prime}(e) \kappa_{j}(e)\right) .
\end{aligned}
$$

The monotonicity of the function $G$ depends on the sign of $\left(\kappa_{j}^{\prime}(e) p_{i}(e)-p_{i}^{\prime}(e) \kappa_{j}(e)\right)$ for all $i, j$. It is thus possible in this context to give rather strong conditions for $G$ to be monotonic and therefore for conclusions (A) or (B) to hold true. For instance, if for all $i, j \in\{1, \ldots, n-1\} e \mapsto \frac{\kappa_{j}(e)}{p_{i}(e)}$ is a non-decreasing function, we can conclude that $G^{\prime}(e)>0$ therefore $G$ is a non-decreasing function of $e$ and statement (B) holds true. This last assumption means that the effort impact is bigger on the distorted probabilities than it is on the probabilities themselves, whatever the risk level (small or catastrophic losses).

\section{Appendices}

\section{A Approximation of the compound Poisson distribution}

In subsection 3.3, as in Section 5, the distribution of the loss random variable $X$ (we temporarily skip the subscript $e$ for simplicity) is given by

$$
P_{X}:=(1-p) \delta_{\{0\}}+p P_{Y},
$$

where $0<p<1, P_{Y}$ denotes the distribution of a positive random variable $Y$, and $\delta_{\{0\}}$ is the Dirac mass at 0 . Let $\lambda>0$ be a real parameter and $P$ be a probability measure on $\mathbb{R}^{+}$. A random variable $\tilde{X}$ which has the compound Poisson distribution $\mathcal{P}(\lambda, P)$ takes the form

$$
\widetilde{X}=\sum_{j=1}^{N} Z_{j}
$$


where $\left(Z_{j}\right)_{j \geq 1}$ is a sequence of independent and identically distributed (i.i.d.) random variables with distribution $P$ and $N$ is a Poisson random variable with parameter $\lambda$, independent of the sequence $\left(Z_{j}\right)_{j \geq 1}$. This model is commonly referred to as the frequency-severity model: the random variable $N$ represents the frequency, i.e. the number of claims in a given time interval (typically one year), and $P$ is the claim severity distribution. Most practical insurance and reinsurance models are given in the form of a compound Poisson distribution ([1]).

In this section of the Appendix, we show that the distribution $P_{X}$ is close to the distribution $\mathcal{P}(\lambda, P)$, for the Kolmogorov distance, with $\lambda=-\log (1-p)$ and $P=P_{Y}$. The approximation is good in the regime where $p$ is close to 0 , which is the case for various practical insurance claims, as natural catastrophes, third party liability, or certain health insurance market segments (see [1], Chapters 2 and 3 and the references therein). The Kolmogorov distance between the probability distributions of the variables $X$ and $\widetilde{X}$ is defined by

$$
d_{K O L}(X, \widetilde{X})=\sup _{x \in \mathbb{R}}|\mathbb{P}(X>x)-\mathbb{P}(\widetilde{X}>x)| .
$$

By computing the characteristic functions, it is not hard to show that both $X_{p}$ and $\widetilde{X}_{p}$ converge in distribution to $\delta_{\{0\}}$ when $p$ goes to 0 . The following Lemma shows how close the distributions of $X$ and $\widetilde{X}$ are with respect to the Kolmogorov distance by providing an explicit upper bound. We write $X_{p}$ and $\widetilde{X}_{p}$ to emphasize the dependence of these variables on the parameter $p$.

Lemma A.1. We have

$$
d_{K O L}\left(X_{p}, \tilde{X}_{p}\right) \leq 2\{p+(1-p) \log (1-p)\}
$$

The right hand side of the above inequality is approximately equal to $10^{-4}$ for $p=1 \%$, and to $10^{-6}$ when $p=$ $0.1 \%$. Figure 4 below shows the error bound function of the previous Lemma, together with an approximation of the Kolmogorov distance between $\widetilde{X}_{p}$ and $X_{p}$, in the case where $X_{p}$ has a distribution given by (A.1), where $P_{Y}$ is the Pareto law with scale parameter $\hat{x}=20$ and shape parameter $k=5$ (see Section 5.1 for details on the Pareto distribution). As mentioned above, the approximation of $\widetilde{X}_{p}$ by $X_{p}$ is good for relatively small values of $p$ : Figure 4 provides the distance and error values for $p$ varying between $0.1 \%$ and $10 \%$. We subdivided the interval $[0.1 \%, 10 \%]$ with a uniform step equal to 0.001 , and for each value of $p$, the cumulative distribution function of $\widetilde{X}_{p}$ has been approximated by the empirical CDF constructed from a set of $10 \times 10^{6}$ simulations of the compound Poisson distribution. This explains the variations in the black curve, coming from the variance of the simulations. 


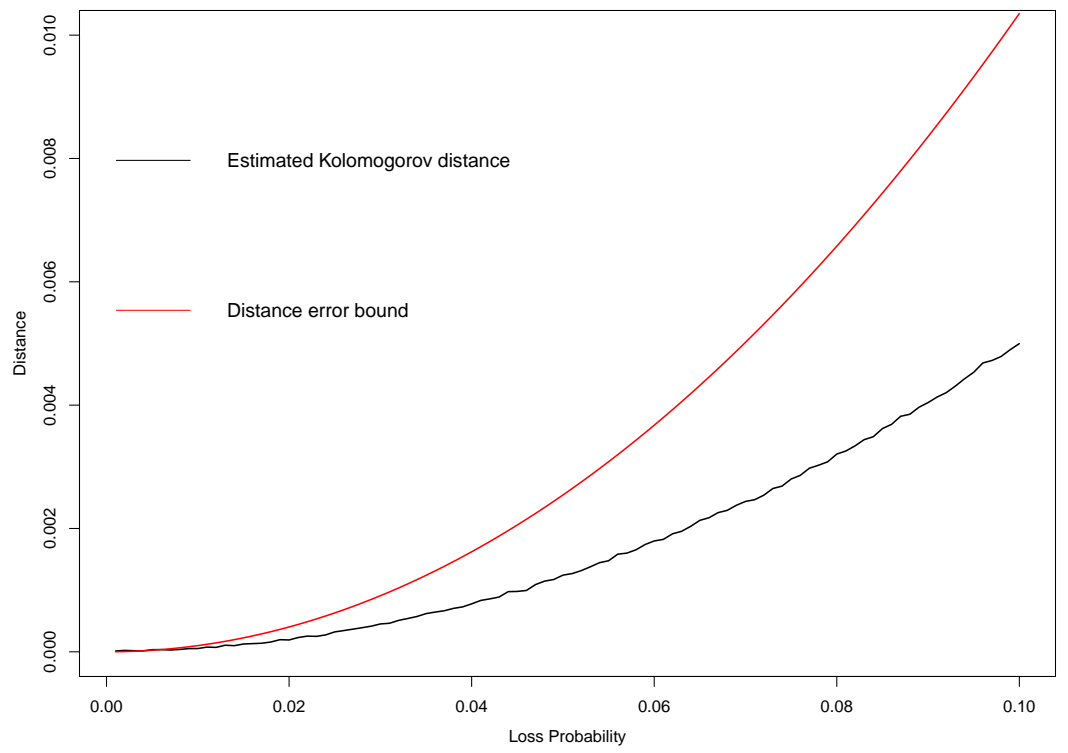

Figure 4: Estimated Kolmogorov distance and error bound from Lemma A.1, as functions of $p$.

Figure 5 below shows both CDFs of $X_{p}$ and $\widetilde{X}_{p}$ in the case of the Pareto distribution described above, for $p=1.98 \%$, i.e. $\lambda=-\log (1-p)=2 \%$. The jump at 0 of the CDF represents the mass at 0 , which is high here $(1-p$ being close to 1$)$. Since the scale parameter $\hat{x}=20$ of the Pareto distribution is also a minimal value, one can observe that the CDFs below are constant in the interval $(0,20)$.

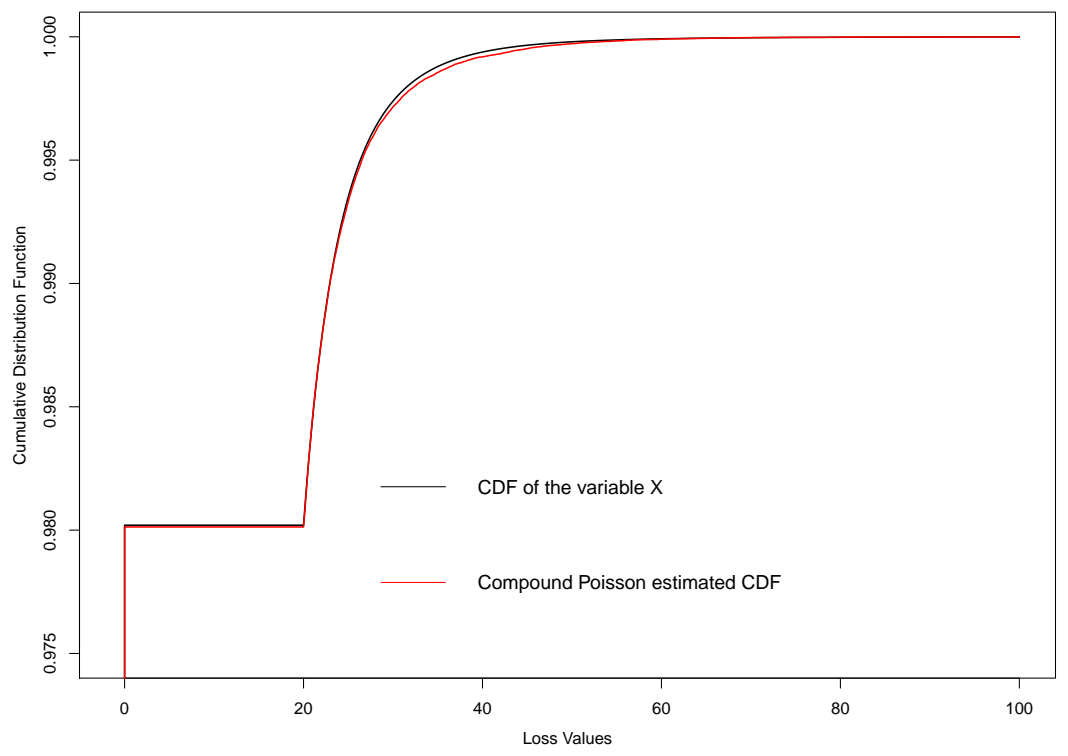

Figure 5: Cumulative distribution functions of $X$ (black curve) and $X_{p}$ (red curve). 
Proof. [of Lemma A.1] On the one hand, by conditioning on $N$, we get for $x \geq 0$,

$$
\begin{aligned}
\mathbb{P}\left(\widetilde{X}_{p}>x\right) & =\sum_{n=0}^{+\infty} \mathbb{P}\left(\sum_{j=1}^{N} Z_{j}>x \mid N=n\right) \mathbb{P}(N=n) \\
& =\lambda \exp (-\lambda) \mathbb{P}\left(Z_{1}>x\right)+\sum_{n=2}^{+\infty} \mathbb{P}\left(\sum_{j=1}^{N} Z_{j}>x \mid N=n\right) \exp (-\lambda) \frac{\lambda^{n}}{n !} .
\end{aligned}
$$

On the other hand, still for $x \geq 0$,

$$
\mathbb{P}\left(X_{p}>x\right)=p \mathbb{P}(Y>x)=(1-\exp (-\lambda)) \mathbb{P}\left(Z_{1}>x\right),
$$

which implies that

$$
\left|\mathbb{P}\left(X_{p}>x\right)-\mathbb{P}\left(\widetilde{X}_{p}>x\right)\right| \leq|\lambda \exp (-\lambda)+\exp (-\lambda)-1|+\sum_{n=2}^{+\infty} \mathbb{P}\left(\sum_{j=1}^{N} Z_{j}>x \mid N=n\right) \exp (-\lambda) \frac{\lambda^{n}}{n !},
$$

and, since both $X_{p}$ and $\tilde{X}_{p}$ are a.s. non-negative,

$$
\begin{aligned}
d_{K O L}\left(X_{p}, \widetilde{X}_{p}\right) & \leq|\lambda \exp (-\lambda)+\exp (-\lambda)-1|+\mathbb{P}(N \geq 2)=2 \mathbb{P}(N \geq 2) \\
& =2(p+(1-p) \log (1-p)) .
\end{aligned}
$$

Remark A.1. If $N$ does not follow the Poisson distribution, then one can still use the same type of approximation as above and prove, using the exact same arguments that

$$
d_{K O L}\left(X_{p}, \tilde{X}_{p}\right) \leq 2 \mathbb{P}(N \geq 2),
$$

where $p=1-\mathbb{P}(N=0)$.

\section{B Computations for self-protection in the Pareto case}

In this section, we explicitly compute the value of $e_{I}(\theta)$. Under Assumption 5.1,

$$
p(e)=\frac{1}{a+e} \text { with } a>1 .
$$

From the characterization (5.14) of $e_{I}(\theta)$, we have

$$
e_{I}(\theta)=-\frac{(1+\theta) \hat{x} k}{k-1} p^{\prime}\left(e_{I}(\theta)\right)=\frac{(1+\theta) \hat{x} k}{k-1} \frac{1}{\left(a+e_{I}(\theta)\right)^{2}}=\frac{c(1+\theta)}{\left(a+e_{I}(\theta)\right)^{2}},
$$

where $c=\frac{\hat{x} k}{k-1}>0$. By rewriting Equation (B.1), we obtain the following equality

$$
e_{I}(\theta)\left(a+e_{I}(\theta)\right)^{2}-c(1+\theta)=0 .
$$

We define the following polynomial function of third degree with coefficients in $\mathbb{R}$

$$
P(X)=X^{3}+2 a X^{2}+a^{2} X-c(1+\theta) .
$$


We can write $P$ as

$$
P(X)=Q\left(X+\frac{2 a}{3}\right)
$$

where $Q(X)=X^{3}-\frac{a^{3}}{3} X-c(1+\theta)-\frac{2 a^{3}}{27}$. Therefore to find to solutions of $P$ we need to determine the solutions of $Q$.

Let $X=x+y$, we have

$$
Q(X)=x^{3}+y^{3}+\left(3 x y-\frac{a^{3}}{3}\right)(x+y)-c(1+\theta)-\frac{2 a^{3}}{27}
$$

where $x^{3}$ and $y^{3}$ are roots of the following polynomial function

$$
R(T)=T^{2}-\left(c(1+\theta)+\frac{2 a^{3}}{27}\right) T+\frac{a^{6}}{27^{2}} .
$$

We denote $\Delta$ the discriminant of $R$ :

$$
D:=27 \Delta=4 a^{6}+27(c(1+\theta))^{2}-32 a^{3} c(1+\theta)-4 a^{6}+36 a^{3} c(1+\theta)=c(1+\theta)\left(27 c(1+\theta)+4 a^{3}\right)>0 .
$$

Therefore $R$ has two distinct solutions in $\mathbb{R}$ given by

$$
\begin{aligned}
& u:=\frac{1}{2}\left(c(1+\theta)+\frac{2 a^{3}}{27}-\sqrt{\frac{4}{27} c(1+\theta) a^{3}+(c(1+\theta))^{2}}\right), \\
& v:=\frac{1}{2}\left(c(1+\theta)+\frac{2 a^{3}}{27}+\sqrt{\frac{4}{27} c(1+\theta) a^{3}+(c(1+\theta))^{2}}\right) .
\end{aligned}
$$

$Q$ has one solution in $\mathbb{R}$ given by $u^{1 / 3}+v^{1 / 3}$. Therefore the solution $e_{I}(\theta) \in \mathbb{R}$ of $P\left(e_{I}(\theta)\right)=0$ is given by

$$
e_{I}(\theta)=t_{1}(\theta)+t_{2}(\theta)-\frac{2 a}{3}
$$

where $t_{1}(\theta)=u^{1 / 3}$ and $t_{2}(\theta)=v^{1 / 3}$.

\section{Proof of Theorem 5.2}

We can rewrite $(5.15)$ as

$$
\inf _{\theta \in\left[0, \theta_{M}\right]}\left\{c_{2} \frac{1}{\left(t_{1}(\theta)+t_{2}(\theta)+\frac{a}{3}\right)^{r_{2}}}-c \frac{1+\theta}{t_{1}(\theta)+t_{2}(\theta)+\frac{a}{3}}\right\},
$$

where $c_{2}:=\frac{r_{2} \hat{x} k}{r_{2} k-1}>0$. We already know that $\theta \mapsto e_{I}(\theta)$ is non decreasing, this implies that $\theta \mapsto$ $\frac{1}{\left(t_{1}(\theta)+t_{2}(\theta)+\frac{a}{3}\right)^{r_{2}}}$ is non increasing. The conclusion of the Theorem will follow from the fact that the function $m$ given by

$$
m(\theta):=\frac{1+\theta}{t_{1}(\theta)+t_{2}(\theta)+\frac{a}{3}},
$$

is an increasing function. By computing the derivative of $m$, we see that it is increasing if and only if

$$
t_{1}(\theta)+t_{2}(\theta)+\frac{a}{3}>(1+\theta)\left(t_{1}^{\prime}(\theta)+t_{2}^{\prime}(\theta)\right)=(1+\theta) \frac{d}{d \theta} e_{I}(\theta) .
$$


To compute $\frac{d}{d \theta} e_{I}(\theta)$, notice that the characterization (5.14) of $e_{I}(\theta)$ can be written as $F\left(\theta, e_{I}(\theta)\right)=0$ with $F(\theta, e):=\frac{1}{c} e(a+e)^{2}-(1+\theta)$. Using the Implicit Function Theorem we get

$$
\begin{aligned}
\frac{d}{d \theta} e_{I}(\theta) & =\frac{c}{3 e_{I}(\theta)^{2}+4 a e_{I}(\theta)+a^{2}}=\frac{c}{3\left(e_{I}(\theta)+a\right)\left(e_{I}(\theta)+\frac{a}{3}\right)} \\
& =\frac{c}{3\left(t_{1}(\theta)+t_{2}(\theta)+\frac{a}{3}\right)\left(t_{1}(\theta)+t_{2}(\theta)-\frac{a}{3}\right)} .
\end{aligned}
$$

After noticing that $\left(t_{1}(\theta)+t_{2}(\theta)-\frac{a}{3}\right)>e_{I}(\theta) \geq 0$, we obtain that (C.1) is equivalent to

$$
\begin{array}{r}
3\left(t_{1}(\theta)+t_{2}(\theta)+\frac{a}{3}\right)^{2}\left(t_{1}(\theta)+t_{2}(\theta)-\frac{a}{3}\right)>(1+\theta) c \\
\Longleftrightarrow 3(x+y)^{3}+a(x+y)^{2}-\frac{a^{2}}{3}(x+y)-\frac{a^{3}}{3^{2}}-c(1+\theta)>0 \\
\Longleftrightarrow 2(x+y)^{3}+a(x+y)^{2}-\left(\frac{a}{3}\right)^{3}+Q(x+y)>0,
\end{array}
$$

where for simplicity, we write $x=t_{1}(\theta), y=t_{2}(\theta)$ and where $Q(X):=X^{3}-\frac{a^{2}}{3} X-c(1+\theta)-2\left(\frac{a}{3}\right)^{3}$. Recall (see Appendix B) that $Q(x+y)=0$, which implies that $2(x+y)^{3}=\frac{2}{3} a^{2}(x+y)+2 c(1+\theta)+4\left(\frac{a}{3}\right)^{3}$. From these observations, we get that the last inequality above is equivalent to

$$
\frac{2}{3} a^{2}(x+y)+2 c(1+\theta)+3\left(\frac{a}{3}\right)^{3}+a(x+y)^{2}>0,
$$

which is satisfied since all the terms are positive. This ends the proof.

\section{References}

[1] Albrecher, H., Teugels, J., And Beirlant, J. Reinsurance: Actuarial and Statistical Aspects. Statistics in Practice. Wiley, 2017.

[2] Artzner, P., Delbaen, F., Eber, J.-M., And Heath, D. Coherent measures of risk. Math. Finance 9, 3 (1999), 203-228.

[3] Athey, S. Monotone comparative statics under uncertainty. The Quarterly Journal of Economics 117, 1 (2002), 187-223.

[4] Barrieu, P., And El Karoui, N. Inf-convolution of risk measures and optimal risk transfer. Finance Stoch. 9, 2 (2005), 269-298.

[5] Bleichrodt, H., And Eeckhoudt, L. Willingness to pay for reductions in health risks when probabilities are distorted. Health Economics 15, 2 (2006), 211-214.

[6] Bowers, N., Gerber, H., Hickman, J., Jones, D., And Nesbitt, C. Actuarial Mathematics. Society of Actuaries, 1997.

[7] Courbage, C. Self-insurance, self-protection and market insurance within the dual theory of choice. The Geneva Risk and Insurance Review 26, 1 (2001), 43-56.

[8] Courbage, C., Loubergé, H., and Peter, R. Optimal prevention for multiple risks. Journal of Risk and Insurance 84, 3 (2017), 899-922. 
[9] Courbage, C., Rey, B., And Treich, N. Prevention and precaution. In Handbook of Insurance, G. Dionne, Ed. Huebner International Series on Risk, Insurance, and Economic Security (New York: Springer), New York, 2013, ch. 8, pp. 185-204.

[10] Cvitanić, J., Possamaï, D., And Touzi, N. Dynamic programming approach to principal-agent problems. arXiv preprint arXiv:1510.07111 (2015).

[11] Cvitanić, J., Wan, X., And Yang, H. Dynamics of contract design with screening. Management Science 59, 5 (2013), 1229-1244.

[12] Cvitanić, J., And Zhang, J. Contract theory in continuous-time models. Springer, 2012.

[13] Deelstra, G., And Plantin, G. Risk theory and reinsurance. Sciences po publications, Sciences Po, 2014.

[14] Doherty, N. A., And Eeckhoudt, L. Optimal insurance without expected utility: The dual theory and the linearity of insurance contracts. Journal of Risk and Uncertainty 10, 2 (Mar 1995), 157-179.

[15] Eeckhoudt, L., Gollier, C., And Schlesinger, H. Economic and Financial Decisions Under Risk. Economic and Financial Decisions Under Risk. Princeton University Press, 2005.

[16] Ehrlich, I., And Becker, G. S. Market insurance, self-insurance, and self-protection. Journal of political Economy 80, 4 (1972), 623-648.

[17] Embrechts, P., And Hofert, M. Risk measures and dependence modeling. In Handbook of Insurance, G. Dionne, Ed. Huebner International Series on Risk, Insurance, and Economic Security (New York: Springer), New York, 2013, ch. 6, pp. 135-166.

[18] Etner, J., ANd Jeleva, M. Risk perception, prevention and diagnostic tests. Health Economics 22, 2 (2013), 144-156.

[19] Föllmer, H., And Schied, A. Stochastic finance: an introduction in discrete time. Walter de Gruyter, 2011.

[20] Föllmer, H., And Schied, A. Stochastic Finance: An Introduction in Discrete Time. De Gruyter Textbook. De Gruyter, 2016.

[21] Frittelli, M., And Rosazza Gianin, E. Putting order in risk measures. Journal of Banking $E$ Finance 26, 7 (July 2002), 1473-1486.

[22] Gollier, C., Hammitt, J. K., And Treich, N. Risk and choice: A research saga. Journal of Risk and Uncertainty 47, 2 (Oct 2013), 129-145.

[23] Hernández Santibáñez, N., Possamaï, D., And Zhou, C. Bank monitoring incentives under moral hazard and adverse selection. arXiv preprint arXiv:1701.05864 (2017).

[24] Hölmstrom, B. Moral hazard and observability. The Bell Journal of Economics 10, 1 (1979), 74-91.

[25] JEWITT, I. Justifying the first-order approach to principal-agent problems. Econometrica 56, 5 (1988), $1177-1190$. 
[26] JEWITT, I. Choosing between risky prospects: The characterization of comparative statics results, and location independent risk. Management Science 35, 1 (1989), 60-70.

[27] Jouini, E., Schachermayer, W., And Touzi, N. Law invariant risk measures have the Fatou property. In Advances in mathematical economics. Vol. 9, vol. 9 of Adv. Math. Econ. Springer, Tokyo, 2006, pp. 49-71.

[28] Jouini, E., Schachermayer, W., And Touzi, N. Optimal risk sharing for law invariant monetary utility functions. Math. Finance 18, 2 (2008), 269-292.

[29] Jullien, B., Salanié, B., And Salanié, F. Should more risk-averse agents exert more effort? The Geneva Papers on Risk and Insurance Theory 24, 1 (Jun 1999), 19-28.

[30] Konrad, K., And Skaperdas, S. Self-insurance and self-protection: A nonexpected utility analysis. The Geneva Risk and Insurance Review 18, 2 (1993), 131-146.

[31] Laeven, R. J., And Goovaerts, M. J. Premium Calculation and Insurance Pricing. American Cancer Society, 2014.

[32] Langlais, E. Willingness to pay for risk reduction and risk aversion without the expected utility assumption. Theory and Decision 59, 1 (Aug 2005), 43-50.

[33] LEE, K. Risk aversion and self-insurance-cum-protection. Journal of Risk and Uncertainty 17, 2 (Nov 1998), 139-151.

[34] Machina, M. Non-expected utility and the robustness of the classical insurance paradigm. The Geneva Risk and Insurance Review 20, 1 (1995), 9-50.

[35] Mastrogiacomo, E., and Rosazza Gianin, E. Pareto optimal allocations and optimal risk sharing for quasiconvex risk measures. Mathematics and Financial Economics 9, 2 (Mar 2015), 149-167.

[36] Meyer, D. J., And Meyer, J. A diamond-stiglitz approach to the demand for self-protection. Journal of Risk and Uncertainty 42, 1 (Feb 2011), 45-60.

[37] Mirrlees, J. The theory of moral hazard and unobservable behaviour: part i (reprint of the unpublished 1975 version). The Review of Economic Studies 66, 1 (1999), 3-21.

[38] Rogerson, W. P. The first-order approach to principal-agent problems. Econometrica 53, 6 (1985), $1357-1367$.

[39] Sannikov, Y. A continuous-time version of the principal-agent problem. The Review of Economic Studies 75, 3 (2008), 957-984.

[40] Shogren, J. F., And Crocker, T. D. Risk, self-protection, and ex ante economic value. Journal of Environmental Economics and Management 20, 1 (1991), 1 - 15.

[41] Touzi, N. Optimal insurance demand under marked point processes shocks. Ann. Appl. Probab. 10, 1 (2000), 283-312. 